\title{
SOME REPRESENTATIONS CONNECTED WITH ULTRAFILTERS AND MAXIMAL LINKED SYSTEMS
}

\author{
Alexander G. Chentsov \\ Krasovskii Institute of Mathematics and Mechanics, \\ Ural Branch of Russian Academy of Sciences, \\ Ekaterinburg, Russia \\ chentsov@imm.uran.ru
}

\begin{abstract}
Ultrafilters and maximal linked systems (MLS) of a lattice of sets are considered. Two following variants of topological equipment are investigated: the Stone and Wallman topologies. These two variants are used both in the case of ultrafilters and for space of MLS. Under Wallman equipment, an analog of superextension is realized. Namely, the space of MLS with topology of the Wallman type is supercompact topological space. By two above-mentioned equipments a bitopological space is realized.
\end{abstract}

Key words: Lattice, Linked system, Ultrafilter.

\section{Introduction}

In connection with the supercompactness property, maximal linked systems (MLS) of closed sets in a topological space (TS) are investigated (see [1-4]; in particular, we note the important statement of [3] about supercompactness of metrizable compactums). The space of «closed» MLS with topology of the Wallman type is a superextension of the initial TS.

Now, following [5], we consider more general approach. Namely, we suppose that a lattice of subsets of arbitrary nonempty set is given. Then, MLS of sets of this lattice are investigated. In particular, the lattice of closed sets in a TS can be used. Then, we obtain the above-mentioned variant of [1-4]. But, many other realizations are possible. For example, we can consider an algebra of sets as variant of the above-mentioned lattice. Note by the way, that in this case the Stone topology on the ultrafilter space is very natural. Since in many respects, MLS are similar to ultrafilters, the Stone equipment is submitted natural and for space of MLS. So, the idea of emploument of the two types of topologies arises: we keep in mind the Wallman and Stone variants.

We recall that ultrafilters were used as generalized elements in problems connected with attainability under constraints of asymptotic character (see, for example, [6-8]). Now, we seek to explore spaces which are comprehending for ultrafilters. In this article, it is established that the space of MLS is comprehending in this sense. In addition, it is logical to consider two characteristic types of topologies both for ultrafilters and for MLS. And what is more, we obtain two bitopological spaces (as a bitopological space, we consider every set equipped with two comparable topologies; in this connection, we note monograph [9]).

The case when two above-mentioned topologies coincide, we consider as degenerate. In the following, characteristic cases of such degeneracy are established (a variant of non-degenerate realization of bitopological space specified also). We indicate important types of lattices for which above-mentioned constructions are realized sufficiently understandably. 


\section{General notions and designations}

We use the standard set-theoretical symbolics (quatifiers, connectives and so on); $\emptyset$ is anempty set and $\triangleq$ is the equality by definition. We call a set by a family in the case when every element of this set is a set also. We take the axiom of choice.

For every objects $x$ and $y$, we denote by $\{x ; y\}$ the set containing $x$ and $y$ and not containing no other elements. If $h$ is an object, then we suppose that $\{h\} \triangleq\{h ; h\}$. Of course, sets are objects. Therefore, by [10, ch. II, §3], for every objects $u$ and $v$, we suppose that $(u, v) \triangleq\{\{u\} ;\{u ; v\}\}$ receiving the ordered pair with first element $u$ and second element $v$. If $z$ is an arbitrary ordered pair, then by $\operatorname{pr}_{1}(z)$ and $\operatorname{pr}_{2}(z)$ we denote the first and second elements of $z$ respectively; of course, $z=\left(\operatorname{pr}_{1}(z), \operatorname{pr}_{2}(z)\right)$ and $\operatorname{pr}_{1}(z)$ and $\operatorname{pr}_{2}(z)$ are defined uniquely.

If $X$ is a set, then by $\mathcal{P}(X)$ we denote the family of all subsets of $X$ and suppose that $\operatorname{Fin}(X)$ is the family of all finite nonempty subsets of $X$; of course. Fin $(X) \subset \mathcal{P}^{\prime}(X)$, where $\mathcal{P}^{\prime}(X) \triangleq \mathcal{P}(X) \backslash\{\emptyset\}$ is the family of all nonempty subsets of $X$. In addition, a family can be used as $X$. For every nonempty family $\mathfrak{X}$, we suppose that

$$
\begin{gathered}
\{\cup\}(\mathfrak{X}) \triangleq\left\{\bigcup_{X \in \mathcal{X}} X: \mathcal{X} \in \mathcal{P}(\mathfrak{X})\right\}, \quad\{\cap\}(\mathfrak{X}) \triangleq\left\{\bigcap_{X \in \mathcal{X}} X: \mathcal{X} \in \mathcal{P}^{\prime}(\mathfrak{X})\right\}, \\
\{\cup\}_{\sharp}(\mathfrak{X}) \triangleq\left\{\bigcup_{X \in \mathcal{K}} X: \mathcal{K} \in \operatorname{Fin}(\mathfrak{X})\right\}, \quad\{\cap\}_{\sharp}(\mathfrak{X}) \triangleq\left\{\bigcap_{X \in \mathcal{K}} X: \mathcal{K} \in \operatorname{Fin}(\mathfrak{X})\right\} ;
\end{gathered}
$$

of course, every family of $(1.1)$ is contained in $\mathcal{P}\left(\bigcup_{X \in \mathfrak{X}} X\right)$ and contains $\mathfrak{X}$. For any set $\mathbb{M}$ and $\mathcal{M} \in \mathcal{P}^{\prime}(\mathcal{P}(\mathbb{M}))$

$$
\mathbf{C}_{\mathbb{M}}[\mathcal{M}] \triangleq\{\mathbb{M} \backslash M: M \in \mathcal{M}\} \in \mathcal{P}^{\prime}(\mathcal{P}(\mathbb{M})) .
$$

In addition (see (1.2)), for any set $S$ and a family $\mathcal{S} \in \mathcal{P}^{\prime}\left(\mathcal{P}(S)\right.$ ), the equality $\mathcal{S}=\mathbf{C}_{S}\left[\mathbf{C}_{S}[\mathcal{S}]\right]$ is realized. If $\mathcal{A}$ is a nonempty family and $B$ is a set, then

$$
\left.\mathcal{A}\right|_{B} \triangleq\{A \cap B: A \in \mathcal{A}\} \in \mathcal{P}^{\prime}(\mathcal{P}(B))
$$

is trace of $\mathcal{A}$ on the set $B$. Usually, in (1.3), the variant $\mathcal{A} \in \mathcal{P}^{\prime}(\mathcal{P}(\mathbb{A}))$ and $B \in \mathcal{P}(\mathbb{A})$, where $\mathbb{A}$ is a set, is considered.

For any sets $A$ and $B$, by $B^{A}$ the set of all mappings from $A$ into $B$ is denoted. Under $f \in B^{A}$ and $a \in A$, by $f(a), f(a) \in B$, the value of $f$ at the point $a$ is denoted. For $f \in B^{A}$ and $C \in \mathcal{P}(A)$, we suppose that $f^{1}(C) \triangleq\{f(x): x \in C\}$; of course, $f^{1}(C) \subset B$ and

$$
(C \neq \emptyset) \Rightarrow\left(f^{1}(C) \neq \emptyset\right) \text {. }
$$

Special families. In given item, we fix a set $I$ (the case $I=\emptyset$ is not excluded). In the form of

$$
\pi[I] \triangleq\left\{\mathcal{I} \in \mathcal{P}^{\prime}(\mathcal{P}(I)) \mid(\emptyset \in \mathcal{I}) \&(I \in \mathcal{I}) \&(A \cap B \in \mathcal{I} \quad \forall A \in \mathcal{I} \quad \forall B \in \mathcal{I})\right\},
$$

we have the family of all $\pi$-systems of subsets of $I$ with «zero» and «unit». In terms of

$$
(\mathrm{LAT})[I] \triangleq\left\{\mathcal{L} \in \mathcal{P}^{\prime}(\mathcal{P}(I)) \mid(\emptyset \in \mathcal{L}) \&(\forall A \in \mathcal{L} \quad \forall B \in \mathcal{L} \quad(A \cup B \in \mathcal{L}) \&(A \cap B \in \mathcal{L}))\right\}
$$

(the family of all lattices of subsets of $I$ ), we define (see (1.4)) the basic family

$$
(\mathrm{LAT})_{0}[I] \triangleq\{\mathcal{I} \in(\mathrm{LAT})[I] \mid I \in \mathcal{I}\}=\{\mathcal{I} \in \pi[I] \mid A \cup B \in \mathcal{I} \quad \forall A \in \mathcal{I} \quad \forall B \in \mathcal{I}\}
$$


of all lattices of subsets of $I$ with «zero» and «unit». In addition, by

$$
\text { (alg) }[I] \triangleq\{\mathcal{A} \in \pi[I] \mid I \backslash A \in \mathcal{A} \quad \forall A \in \mathcal{A}\}
$$

the family of all algebras of subsets of $I$ is defined. Moreover, by

$$
\text { (top) }[I] \triangleq\left\{\tau \in \pi[I] \mid \bigcup_{G \in \mathcal{G}} G \in \tau \quad \forall \mathcal{G} \in \mathcal{P}^{\prime}(\tau)\right\}=\left\{\tau \in(\mathrm{LAT})_{0}[I] \mid \bigcup_{G \in \mathcal{G}} G \in \tau \quad \forall \mathcal{G} \in \mathcal{P}^{\prime}(\tau)\right\}
$$

and (analogously)

$$
\begin{gathered}
(\operatorname{clos})[I] \triangleq\left\{\mathcal{F} \in \mathcal{P}^{\prime}(\mathcal{P}(I)) \mid(\emptyset \in \mathcal{F}) \&(I \in \mathcal{F}) \&(A \cup B \in \mathcal{F} \quad \forall A \in \mathcal{F} \quad \forall B \in \mathcal{F}) \&\right. \\
\left.\left(\bigcap_{F \in \mathcal{F}^{\prime}} F \in \mathcal{F} \quad \forall \mathcal{F}^{\prime} \in \mathcal{P}^{\prime}(\mathcal{F})\right)\right\}=\left\{\mathcal{F} \in(\mathrm{LAT})_{0}[I] \mid \bigcap_{F \in \mathcal{F}^{\prime}} F \in \mathcal{F} \quad \forall \mathcal{F}^{\prime} \in \mathcal{P}^{\prime}(\mathcal{F})\right\}
\end{gathered}
$$

we define the families of all open and closed [11] topologies on $I$ respectively. So, by (1.7)- (1.9) we obtain many useful examples of lattices of the family (1.6). Yet one particular case of a lattice of subsets of $I$ is connected with $\sigma$-topologies of A.D. Alexandroff [12]:

$$
(\sigma-\text { top })[I] \triangleq\left\{\tau \in \pi[I] \mid \bigcup_{k \in \mathbb{N}} G_{k} \in \tau \quad \forall\left(G_{k}\right)_{k \in \mathbb{N}} \in \tau^{\mathbb{N}}\right\} \subset(\mathrm{LAT})_{0}[I]
$$

where as usually $\mathbb{N} \triangleq\{1 ; 2 ; \ldots\}$. Of course, under $\mathcal{A} \in(\operatorname{alg})[I]$, in the form of $(I, \mathcal{A})$, we obtain a measurable space with algebra of sets. If $\tau \in(\operatorname{top})[I]$, then $(I, \tau)$ is a topological space (TS). In addition, we use the notions $T_{1^{-}}$and $T_{2}$-space (see [13, Ch.1]). Moreover, we use compactness [13, Ch.3] and other notions relating to general topology; see [13]. In particular, under $\tau \in($ top $)[I]$, by $(\tau-$ comp $)[I]$ the family of all compact in $(I, \tau)$ subsets of $I$ is denoted; $(\tau-\operatorname{comp})[I] \in \mathcal{P}^{\prime}(\mathcal{P}(I))$. We note the obvious property

$$
\mathcal{L} \cup\{I\} \in(\mathrm{LAT})_{0}[I] \quad \forall \mathcal{L} \in(\mathrm{LAT})[I] .
$$

Of course, in (1.10) we have an insignificant transformation of initial lattice.

Let

$$
\widetilde{\pi}^{0}[I] \triangleq\{\mathcal{L} \in \pi[I] \mid \forall L \in \mathcal{L} \quad \forall x \in I \backslash L \quad \exists \Lambda \in \mathcal{L}:(x \in \Lambda) \&(\Lambda \cap L=\emptyset)\} .
$$

Moreover, let

$$
(\mathrm{Cen})[\mathcal{L}] \triangleq\left\{\mathcal{Z} \in \mathcal{P}^{\prime}(\mathcal{L}) \mid \bigcap_{Z \in \mathcal{K}} Z \neq \emptyset \quad \forall \mathcal{K} \in \operatorname{Fin}(\mathcal{Z})\right\} \quad \forall \mathcal{L} \in \pi[I]
$$

Bases and subbases. For brevity of desingnations, until end of this section, we fix a nonempty set $X$ and use (1.1). Then,

$$
\begin{gathered}
(\mathrm{BAS})[X] \triangleq\left\{\mathcal{B} \in \mathcal{P}^{\prime}(\mathcal{P}(X)) \mid\left(X=\bigcup_{B \in \mathcal{B}} B\right) \&\left(\forall B_{1} \in \mathcal{B} \quad \forall B_{2} \in \mathcal{B}\right.\right. \\
\left.\left.\forall x \in B_{1} \cap B_{2} \exists B_{3} \in \mathcal{B}:\left(x \in B_{3}\right) \&\left(B_{3} \subset B_{1} \cap B_{2}\right)\right)\right\}
\end{gathered}
$$

is the family of all open bases of topologies on $X$. Under $\mathcal{B} \in(\mathrm{BAS})[X]$, we obtain that $\{\cup\}(\mathcal{B}) \in$ (top) $[X]$. Then, for $\tau \in($ top $)[X]$

$$
(\tau-\mathrm{BAS})_{0}[X] \triangleq\{\mathcal{B} \in(\mathrm{BAS})[X] \mid \tau=\{\cup\}(\mathcal{B})\}
$$


is the family of all bases of TS $(X, \tau)$. In addition,

$$
(\mathrm{p}-\mathrm{BAS})[X] \triangleq\left\{\mathfrak{X} \in \mathcal{P}^{\prime}(\mathcal{P}(X)) \mid\{\cap\}_{\sharp}(\mathfrak{X}) \in(\mathrm{BAS})[X]\right\}=\left\{\mathfrak{X} \in \mathcal{P}^{\prime}(\mathcal{P}(X)) \mid X=\bigcup_{\mathbb{X} \in \mathfrak{X}} \mathbb{X}\right\}
$$

is the family of all open subbases of topologies on $X$. For any $\mathfrak{X} \in(\mathrm{p}-\mathrm{BAS})[X]$, we obtain that $\{\cup\}\left(\{\cap\}_{\sharp}(\mathfrak{X})\right) \in($ top $)[X]$. Finally, under $\tau \in($ top $)[X]$, we suppose that

$$
(\mathrm{p}-\mathrm{BAS})_{0}[X ; \tau] \triangleq\left\{\mathfrak{X} \in(\mathrm{p}-\mathrm{BAS})[X] \mid\{\cap\}_{\sharp}(\mathfrak{X}) \in(\tau-\mathrm{BAS})_{0}[X]\right\} ;
$$

so, we obtain the family of all open subbases of TS $(X, \tau)$. It is useful to introduce one auxiliary construction of [6]:

$$
(\mathrm{op}-\mathrm{BAS})_{\emptyset}[X] \triangleq\{\mathcal{B} \in(\mathrm{BAS})[X] \mid \emptyset \in \mathcal{B}\} ;
$$

moreover, it is logical to consider the following family:

$$
(\mathrm{p}-\mathrm{BAS})_{\emptyset}[X] \triangleq\left\{\mathfrak{X} \in(\mathrm{p}-\mathrm{BAS})[X] \mid\{\cap\}_{\sharp}(\mathfrak{X}) \in(\mathrm{op}-\mathrm{BAS})_{\emptyset}[X]\right\} .
$$

If $\tau \in($ top $)[X]$, then we suppose that

$$
\begin{gathered}
(\mathrm{p}-\mathrm{BAS})_{\emptyset}^{0}[X ; \tau] \triangleq\left\{\mathcal{X} \in(\mathrm{p}-\mathrm{BAS})_{0}[X ; \tau] \mid \emptyset \in\{\cap\}_{\sharp}(\mathcal{X})\right\}, \\
(\mathrm{p}-\mathrm{BAS})_{\emptyset}^{0}[X ; \tau] \subset(\mathrm{p}-\mathrm{BAS})_{\emptyset}[X] .
\end{gathered}
$$

Of course, under $\mathcal{B} \in(\mathrm{BAS})[X]$, we obtain that $\mathcal{B} \cup\{\emptyset\} \in(\text { op }-\mathrm{BAS})_{\emptyset}[X]$ and $\{\cup\}(\mathcal{B} \cup\{\emptyset\})=$ $\{\cup\}(\mathcal{B})$. So, we were introduce an unessential transformation of open bases; the goal of such transformation was indicated in $[6, \S 1]$.

Now, we consider closed bases and subbases. Let

$$
\begin{gathered}
(\mathrm{cl}-\mathrm{BAS})[X] \triangleq\left\{\mathcal{B} \in \mathcal{P}^{\prime}(\mathcal{P}(X)) \mid(X \in \mathcal{B}) \&\left(\bigcap_{B \in \mathcal{B}} B=\emptyset\right) \&\right. \\
\left.\&\left(\forall B_{1} \in \mathcal{B} \quad \forall B_{2} \in \mathcal{B} \quad \forall x \in X \backslash\left(B_{1} \cup B_{2}\right) \quad \exists B_{3} \in \mathcal{B}:\left(B_{1} \cup B_{2} \subset B_{3}\right) \&\left(x \notin B_{3}\right)\right)\right\} ;
\end{gathered}
$$

so, we introduce the family of all closed bases of topologies on $X$. Of course, $\{\cap\}(\mathfrak{B}) \in($ clos $)[X]$ for $\mathfrak{B} \in(\mathrm{cl}-\mathrm{BAS})[X]$. Under $\tau \in($ top $)[X]$, we suppose that

$$
(\mathrm{cl}-\mathrm{BAS})_{0}[X ; \tau] \triangleq\left\{\mathcal{B} \in(\mathrm{cl}-\mathrm{BAS})[X] \mid \mathbf{C}_{X}[\tau]=\{\cap\}(\mathcal{B})\right\} ;
$$

then, the family of all closed bases of TS $(X, \tau)$ is defined. Now, we introduce the family of all closed subbases of topologies on $X$ :

$$
(\mathrm{p}-\mathrm{BAS})_{\mathrm{cl}}[X] \triangleq\left\{\mathcal{X} \in \mathcal{P}^{\prime}(\mathcal{P}(X)) \mid\{\cup\}_{\sharp}(\mathcal{X}) \in(\mathrm{cl}-\mathrm{BAS})[X]\right\} .
$$

Respectively, in the form

$$
(\mathrm{p}-\mathrm{BAS})_{\mathrm{cl}}^{0}[X ; \tau] \triangleq\left\{\mathcal{X} \in(\mathrm{p}-\mathrm{BAS})_{\mathrm{cl}}[X] \mid\{\cup\}_{\sharp}(\mathcal{X}) \in(\mathrm{cl}-\mathrm{BAS})_{0}[X ; \tau]\right\},
$$

we obtain the family of all closed subbases of TS $(X, \tau)$. In addition,

$$
\begin{aligned}
& \left(\{\cup\}\left(\{\cap\}_{\sharp}(\mathfrak{S})\right) \in(\text { top })[X] \quad \forall \mathfrak{S} \in(\mathrm{p}-\mathrm{BAS})[X]\right) \& \\
& \left(\{\cap\}\left(\{\cup\}_{\sharp}(\mathcal{S})\right) \in(\operatorname{clos})[X] \quad \forall \mathcal{S} \in(\mathrm{p}-\mathrm{BAS})_{\mathrm{cl}}[X]\right) .
\end{aligned}
$$


Recall following useful duality relations:

$$
\begin{gathered}
\left(\mathbf{C}_{X}[\mathcal{B}] \in(\text { op }-\mathrm{BAS})_{\emptyset}[X] \quad \forall \mathcal{B} \in(\mathrm{cl}-\mathrm{BAS})[X]\right) \&\left(\mathbf{C}_{X}[\mathfrak{B}] \in(\mathrm{cl}-\mathrm{BAS})[X]\right. \\
\left.\forall \mathfrak{B} \in(\mathrm{op}-\mathrm{BAS})_{\emptyset}[X]\right) .
\end{gathered}
$$

We note also $[6,(1.20)]$ and some simple corollaries of $[6,(1.17)]$ :

$$
\begin{gathered}
\left(\mathbf{C}_{X}[\{\cap\}(\mathcal{B})]=\{\cup\}\left(\mathbf{C}_{X}[\mathcal{B}]\right) \in(\text { top })[X] \quad \forall \mathcal{B} \in(\mathrm{cl}-\mathrm{BAS})[X]\right) \&\left(\{\cap\}\left(\mathbf{C}_{X}[\mathfrak{B}]\right)=\right. \\
\left.\mathbf{C}_{X}[\{\cup\}(\mathfrak{B})] \in(\operatorname{clos})[X] \quad \forall \mathfrak{B} \in(\text { op }-\mathrm{BAS})_{\emptyset}[X]\right) .
\end{gathered}
$$

In connection with (1.12) and (1.13), it is useful to note that under $\beta \in(\mathrm{BAS})[X]$

$$
\beta \cup\{\emptyset\} \in(\text { op }-\mathrm{BAS})_{\emptyset}[X]:\{\cup\}(\beta)=\{\cup\}(\beta \cup\{\emptyset\}) .
$$

Now, consider some analogs concerning to subbases. In particular,

$$
(\mathrm{p}-\mathrm{BAS})_{\emptyset}[X]=\left\{\mathfrak{X} \in(\mathrm{p}-\mathrm{BAS})[X] \mid \emptyset \in\{\cap\}_{\sharp}(\mathfrak{X})\right\} .
$$

In terms of (1.14), we obtain the next analog of (1.12):

$$
\begin{gathered}
\left(\mathbf{C}_{X}[\mathfrak{X}] \in(\mathrm{p}-\mathrm{BAS})_{\emptyset}[X] \quad \forall \mathfrak{X} \in(\mathrm{p}-\mathrm{BAS})_{\mathrm{cl}}[X]\right) \& \\
\left(\mathbf{C}_{X}[\mathcal{X}] \in(\mathrm{p}-\mathrm{BAS})_{\mathrm{cl}}[X] \quad \forall \mathcal{X} \in(\mathrm{p}-\mathrm{BAS})_{\emptyset}[X]\right) .
\end{gathered}
$$

As a corollary, from (1.15), it follows that $\forall \tau \in($ top $)[X]$

$$
\begin{gathered}
\left(\mathbf{C}_{X}[\mathfrak{X}] \in(\mathrm{p}-\mathrm{BAS})_{\emptyset}^{0}[X ; \tau] \quad \forall \mathfrak{X} \in(\mathrm{p}-\mathrm{BAS})_{\mathrm{cl}^{0}}^{0}[X ; \tau]\right) \& \\
\left(\mathbf{C}_{X}[\mathcal{X}] \in(\mathrm{p}-\mathrm{BAS})_{\mathrm{cl}}^{0}[X ; \tau] \quad \forall \mathcal{X} \in(\mathrm{p}-\mathrm{BAS})_{\emptyset}^{0}[X ; \tau]\right) .
\end{gathered}
$$

A special family of lattices. By (1.10) we can consider lattices from (LAT) $[X]$. Now, we introduce the family

$$
(\downarrow-\mathrm{LAT})^{0}[X] \triangleq\left\{\mathcal{L} \in(\mathrm{LAT})[X] \mid(X \notin \mathcal{L}) \&(\{x\} \in \mathcal{L} \quad \forall x \in X) \&\left(\bigcap_{L \in \mathcal{L}^{\prime}} L \in \mathcal{L} \quad \forall \mathcal{L}^{\prime} \in \mathcal{P}^{\prime}(\mathcal{L})\right)\right\}
$$

It is possible to consider elements of (1.17) as lattices of «small» subsets of $X$. It is obvious that

$$
\mathcal{L} \cup\{X\} \in(\operatorname{clos})[X] \quad \forall \mathcal{L} \in(\downarrow-\mathrm{LAT})^{0}[X] .
$$

The relation (1.18) assumes an amplification. For this, we introduce

$$
\begin{gathered}
\left((\mathcal{D}-\text { top })[X] \triangleq\left\{\tau \in(\text { top })[X] \mid\{x\} \in \mathbf{C}_{X}[\tau] \quad \forall x \in X\right\}\right) \& \\
((\mathcal{D}-\operatorname{clos})[X] \triangleq\{\mathcal{F} \in(\operatorname{clos})[X] \mid\{x\} \in \mathcal{F} \quad \forall x \in X\}) ;
\end{gathered}
$$

of course, under $\mathbf{t} \in(\mathcal{D}-$ top $)[X]$, in the form of $(X, \mathbf{t})$, we have a $T_{1}$-space. In addition, open and closed topologies from (1.19) are situated in the natural duality. From (1.17) and (1.19), we obtain that

$$
\mathcal{L} \cup\{X\} \in(\mathcal{D}-\operatorname{clos})[X] \quad \forall \mathcal{L} \in(\downarrow-\mathrm{LAT})^{0}[X] .
$$

So, under $\mathcal{L} \in(\downarrow-\mathrm{LAT})^{0}[X]$, we obtain that

$$
\tau_{\mathcal{L}}^{0}[X] \triangleq \mathbf{C}_{X}[\mathcal{L} \cup\{X\}]=\mathbf{C}_{X}[\mathcal{L}] \cup\{\emptyset\} \in(\mathcal{D}-\text { top })[X]
$$


realizes the initial lattice $\mathcal{L} \cup\{X\}$ as the lattice $\mathbf{C}_{X}\left[\tau_{\mathcal{L}}^{0}[X]\right]$ of closed sets in $T_{1}$-space:

$$
\mathcal{L} \cup\{X\}=\mathbf{C}_{X}\left[\tau_{\mathcal{L}}^{0}[X]\right]
$$

in addition, $\left(X, \tau_{\mathcal{L}}^{0}[X]\right)$ is not $T_{2}$-space and

$$
\tau_{\mathcal{L}}^{0}[X] \neq \mathcal{P}(X) .
$$

Recall that $\mathcal{L} \cup\{X\} \in(\mathrm{LAT})_{0}[X] \forall \mathcal{L} \in(\downarrow-\mathrm{LAT})^{0}[X]$. Now, we consider some examples.

Example 1.1. Suppose that $X$ is equipped with a pseudometric

$$
\rho: X \times X \rightarrow[0, \infty[
$$

(here, $[0, \infty[\triangleq\{\xi \in \mathbb{R} \mid 0 \leqslant \xi\}$, where $\mathbb{R}$ is real line); so, $(X, \rho)$ is a pseudometric space, $X \neq \emptyset$. Let

$$
B_{\rho}(X, \varepsilon) \triangleq\{y \in X \mid \rho(x, y) \leqslant \varepsilon\} \quad \forall x \in X \quad \forall \varepsilon \in[0, \infty[.
$$

We suppose that

$$
\begin{gathered}
\mathfrak{B}^{\sharp}(X, \rho) \triangleq\left\{H \in \mathcal{P}(X) \mid \exists x \in X \exists \varepsilon \in\left[0, \infty\left[: H \subset B_{\rho}(x, \varepsilon)\right\}=\right.\right. \\
=\{H \in \mathcal{P}(X) \mid \exists x \in X \exists \varepsilon \in] 0, \infty\left[: H \subset B_{\rho}(x, \varepsilon)\right\},
\end{gathered}
$$

where $] 0, \infty\left[\triangleq\{\xi \in \mathbb{R} \mid 0<\xi\}\right.$. Of course, $\mathfrak{B}^{\sharp}(X, \rho)$ is the family of $\rho$-bounded subsets of $X$.

We suppose that $X \notin \mathfrak{B}^{\sharp}(X, \rho)$. So, the pseudometric $\rho$ is unbounded (in particular, real line $\mathbb{R}$ with the metric-modulus can be used as $(X, \rho))$. Then,

$$
\mathfrak{B}^{\sharp}(X, \rho) \in(\downarrow-\mathrm{LAT})^{0}[X] .
$$

The proof of (1.23) is obvions (see (1.17)). We note only that $\mathfrak{B}^{\sharp}(X, \rho)=\{\cap\}\left(\mathfrak{B}^{\sharp}(X, \rho)\right)$.

Example 1.2. Fix a topology $\tau \in($ top $)[X]$ for which $(X, \tau)$ is a $T_{2}$-space (of course, $X \neq \emptyset$ ). We suppose that

$$
X \notin(\tau-\operatorname{comp})[X] .
$$

So, $T_{2}$-space $(X, \tau)$ is noncompact. Then

$$
(\tau-\operatorname{comp})[X] \in(\downarrow-\mathrm{LAT})^{0}[X] .
$$

We consider the scheme of the proof of (1.24). In addition, we recall some known properties. So, at first, we show that

$$
(\tau-\operatorname{comp})[X] \in(\mathrm{LAT})[X]
$$

(we check this understandable property). We recall that $\emptyset \in(\tau-\operatorname{comp})[X]$ and

$$
\{x\} \in(\tau-\operatorname{comp})[X] \quad \forall x \in X .
$$

So, $(\tau-\operatorname{comp})[X] \in \mathcal{P}^{\prime}(\mathcal{P}(X))$. Let $\mathbb{A} \in(\tau-\operatorname{comp})[X]$ and $\mathbb{B} \in(\tau-\operatorname{comp})[X]$. Then $\mathbb{A} \cup \mathbb{B} \in$ $(\tau-$ comp $)[X]$ by definition of the compactness property. Consider $\mathbb{A} \cap \mathbb{B}$. By separability of $(X, \tau)$ we have that

$$
\left(\mathbb{A} \in \mathbf{C}_{X}[\tau]\right) \&\left(\mathbb{B} \in \mathbf{C}_{X}[\tau]\right)
$$


as a corollary, $\mathbb{A} \cap \mathbb{B} \in \mathbf{C}_{X}[\tau]$. If $\left.\theta \triangleq \tau\right|_{\mathbb{A}}$, then by transitivity of the operation of passage to a subspace of TS we have the equality

$$
\left.\tau\right|_{\mathbb{A} \cap \mathbb{B}}=\left.\theta\right|_{\mathbb{A} \cap \mathbb{B}},
$$

where $(\mathbb{A}, \theta)$ is a compact TS. By (1.27) $\mathbb{A} \cap \mathbb{B} \in \mathbf{C}_{\mathbb{A}}[\theta]$ and, as a corollary,

$$
\mathbb{A} \cap \mathbb{B} \in(\theta-\operatorname{comp})[\mathbb{A}] .
$$

So, $\left(\mathbb{A} \cap \mathbb{B},\left.\theta\right|_{\mathbb{A} \cap \mathbb{B}}\right)$ is a compact TS. Using (1.28), we obtain that $\left(\mathbb{A} \cap \mathbb{B},\left.\tau\right|_{\mathbb{A} \cap \mathbb{B}}\right)$ is a compact TS. Therefore, $\mathbb{A} \cap \mathbb{B} \in(\tau-$ comp $)[X]$. Since the choice of $\mathbb{A}$ and $\mathbb{B}$ was arbitrary, it is established that $\forall A \in(\tau-\operatorname{comp})[X] \quad \forall B \in(\tau-\operatorname{comp})[X]$

$$
(A \cup B \in(\tau-\operatorname{comp})[X]) \&(A \cap B \in(\tau-\operatorname{comp})[X]) .
$$

So, by (1.5) and (1.29) we obtain that $(\tau-\operatorname{comp})[X] \in(\mathrm{LAT})[X]$. We recall (1.26). Finally, let $\mathcal{T} \in \mathcal{P}^{\prime}((\tau-$ comp $)[X])$. Then, in particular, $\mathcal{T} \in \mathcal{P}^{\prime}(\mathcal{P}(X))$ and we have the set

$$
\mathbb{T} \triangleq \bigcap_{T \in \mathcal{T}} T \in \mathcal{P}(X)
$$

By separability of $(X, \tau) \mathcal{T} \subset \mathbf{C}_{X}[\tau]$ and (see (1.30)) $\mathbb{T} \in \mathbf{C}_{X}[\tau]$. In addition, $\mathcal{T} \neq \emptyset$. Choose $\mathbf{T} \in \mathcal{T}$; then $\mathbf{T} \in(\tau-$ comp $)[X]$ and $\mathbb{T} \in \mathcal{P}(\mathbf{T})$. We note that $\left.\mathbf{t} \triangleq \tau\right|_{\mathbf{T}} \in($ top $)[\mathbf{T}]$ and TS $(\mathbf{T}, \mathbf{t})$ is a compactum. In addition, $\mathbb{T} \in \mathbf{C}_{\mathbf{T}}[\mathbf{t}]$ (indeed, $(\mathbf{T}, \mathbf{t})$ is a closed subspace of $(X, \tau)$ ). As a corollary, $\mathbb{T} \in(\mathbf{t}-$ comp $)[\mathbf{T}]$; therefore, $\left.\mathbf{t}\right|_{\mathbb{T}}$ realizes compactum $\left(\mathbb{T},\left.\mathbf{t}\right|_{\mathbb{T}}\right)$. But, by transitivity we obtain that $\left.\mathbf{t}\right|_{\mathbb{T}}=\left.\tau\right|_{\mathbb{T}}$. So, $\left(\mathbb{T},\left.\tau\right|_{\mathbb{T}}\right)$ is compactum; as a corollary $\mathbb{T} \in(\tau-$ comp $)[X]$. Since the choice of $\mathcal{T}$ was arbitrary, we establish (see (1.30)) that

$$
\bigcap_{K \in \mathcal{C}} K \in(\tau-\text { comp })[X] \quad \forall \mathcal{C} \in \mathcal{P}^{\prime}((\tau-\text { comp })[X]) .
$$

Therefore (see (1.17), (1.26), (1.29), and (1.31)), we obtain (1.24).

Example 1.3. Consider the case of infinite set $X$ and suppose that $($ FIN $)[X] \triangleq \operatorname{Fin}(X) \cup\{\emptyset\}$ (the family of all finite subsets of $X$.) Of course, in our case

$$
X \notin(\mathrm{FIN})[X] .
$$

We show that $(\mathrm{FIN})[X] \in(\downarrow-\mathrm{LAT})^{0}[X]$. Indeed, $(\mathrm{FIN})[X] \in(\mathrm{LAT})[X]$ by obvious properties of finite sets. Moreover, $\{x\} \in(\mathrm{FIN})[X] \quad \forall x \in X$. Let $\mathcal{F} \in \mathcal{P}^{\prime}((\mathrm{FIN})[X])$. Then, $\mathcal{F} \neq \emptyset$ and $\mathcal{F} \subset($ FIN $)[X]$. We choose $\mathbb{F} \in \mathcal{F}$. Then, in particular, $\mathbb{F} \in($ FIN $)[X]$. Since

$$
\mathbf{F} \triangleq \bigcap_{F \in \mathcal{F}} F \subset \mathbb{F}
$$

we have the obvious inclusion $\mathbf{F} \in(\mathrm{FIN})[X]$. Since the choice of $\mathcal{F}$ was arbitrary, we obtain that

$$
\bigcap_{H \in \mathcal{H}} H \in(\mathrm{FIN})[X] \quad \forall \mathcal{H} \in \mathcal{P}^{\prime}((\mathrm{FIN})[X]) .
$$

So, the required property $(\mathrm{FIN})[X] \in(\downarrow-\mathrm{LAT})^{0}[X]$ is established. Now, we note that

$$
\tau_{(\mathrm{FIN})[X]}^{0}[X]=\mathbf{C}_{X}[(\mathrm{FIN})[X]] \cup\{\emptyset\} \in(\text { top })[X]
$$


is known cofinite topology and

$$
(\mathrm{FIN})[X] \cup\{X\}=\mathbf{C}_{X}\left[\tau_{(\mathrm{FIN})[X]}^{0}[X]\right]
$$

is the family of closed sets in this topology.

Example 1.4. Let $X, X \neq \emptyset$, be uncountable set. Consider the family $\omega[X]$ of all no more than countable subsets of $X$. In addition, $\omega[X]=($ count $)[X] \cup\{\emptyset\}$, where (count) $[X] \triangleq\left\{f^{1}(\mathbb{N}): f \in X^{\mathbb{N}}\right\}$ under $\mathbb{N}=\{1 ; 2 ; \ldots\}$ and $\widetilde{f}^{1}(\mathbb{N})=\{\widetilde{f}(k): k \in \mathbb{N}\}$ for $\widetilde{f} \in X^{\mathbb{N}}$. Then, $\omega[X] \in(\downarrow-\text { LAT })^{0}[X]$. The corresponding proof is similar to previous example.

Coverings and linked families. Recall that $X$ is a nonempty set. If $\mathcal{X} \in \mathcal{P}^{\prime}(\mathcal{P}(X))$, then

$$
(\mathrm{COV})[X \mid \mathcal{X}] \triangleq\left\{\mathfrak{X} \in \mathcal{P}^{\prime}(\mathcal{X}) \mid X=\bigcup_{\mathbb{X} \in \mathfrak{X}} \mathbb{X}\right\}
$$

is the family of all coverings of $X$ by sets from $\mathcal{X}$. Let

$$
(\text { link })[X] \triangleq\left\{\mathcal{X} \in \mathcal{P}^{\prime}(\mathcal{P}(X)) \mid A \cap B \neq \emptyset \quad \forall A \in \mathcal{X} \quad \forall B \in \mathcal{X}\right\}
$$

Then, the family of all linked systems of subsets of $X$ is introduced. Moreover, suppose that

$$
(\text { link })_{0}[X] \triangleq\{\mathcal{E} \in(\text { link })[X] \mid \forall \mathcal{S} \in(\text { link })[X] \quad(\mathcal{E} \subset \mathcal{S}) \Rightarrow(\mathcal{E}=\mathcal{S})\} .
$$

We obtain the family of all MLS of subsets of $X$. In the following, we consider MLS containing in a given family. So, under $\mathfrak{X} \in \mathcal{P}^{\prime}(\mathcal{P}(X))$

$$
(\mathfrak{X}-\text { link })[X] \triangleq\{\mathcal{E} \in(\text { link })[X] \mid \mathcal{E} \subset \mathfrak{X}\} \in \mathcal{P}((\text { link })[X])
$$

and by analogy with (1.34)

$$
(\mathfrak{X}-\operatorname{link})_{0}[X] \triangleq\{\mathcal{E} \in(\mathfrak{X}-\operatorname{link})[X] \mid \forall \widetilde{\mathcal{E}} \in(\mathfrak{X}-\operatorname{link})[X] \quad(\mathcal{E} \subset \widetilde{\mathcal{E}}) \Rightarrow(\mathcal{E}=\widetilde{\mathcal{E}})\} .
$$

In (1.36), we obtain the family of all MLS containing in the family $\mathfrak{X}$.

Proposition 1. If $\mathfrak{X} \in \mathcal{P}^{\prime}(\mathcal{P}(X)), \mathcal{E} \in($ link $)[X]$, and $\mathfrak{X} \cap \mathcal{E} \neq \emptyset$, then

$$
\mathfrak{X} \cap \mathcal{E} \in(\mathfrak{X}-\operatorname{link})[X] .
$$

P r o o f. Fix $\mathfrak{X}$ and $\mathcal{E}$ with above-mentioned properties. In particular, $\mathfrak{X} \cap \mathcal{E} \in \mathcal{P}^{\prime}(\mathcal{P}(X))$. Let $U \in \mathfrak{X} \cap \mathcal{E}$ and $V \in \mathfrak{X} \cap \mathcal{E}$. Then, in particular, $U \in \mathcal{E}$ and $V \in \mathcal{E}$. By (1.33) we obtain that $U \cap V \neq \emptyset$. Since the choice of $U$ and $V$ was arbitrary, we have the property

$$
\mathfrak{X} \cap \mathcal{E} \in \mathcal{P}^{\prime}(\mathcal{P}(X)): A \cap B \neq \emptyset \quad \forall A \in \mathfrak{X} \cap \mathcal{E} \quad \forall B \in \mathfrak{X} \cap \mathcal{E} .
$$

By (1.33) $\mathfrak{X} \cap \mathcal{E} \in($ link $)[X]$. Then (see (1.35)), (1.37) is fulfilled.

Supercompactness. If $\tau \in($ top $)[X]$, then we suppose that

$$
((\mathrm{p}, \text { bin })-\mathrm{cl})[X ; \tau] \triangleq\left\{\mathfrak{X} \in(\mathrm{p}-\mathrm{BAS})_{\mathrm{cl}}^{0}[X ; \tau] \mid \bigcap_{\mathbb{X} \in \mathcal{X}} \mathbb{X} \neq \emptyset \quad \forall \mathcal{X} \in(\mathfrak{X}-\operatorname{link})[X]\right\}
$$


$((1.38)$ is the family of all closed binary subbases of $\operatorname{TS}(X, \tau))$; it is obvious that $\forall \kappa \in(\mathrm{p}-\mathrm{BAS})_{\mathrm{cl}}^{0}[X ; \tau]$

$$
(\kappa \in((\mathrm{p}, \mathrm{bin})-\mathrm{cl})[X ; \tau]) \Leftrightarrow\left(\forall \mathfrak{C} \in(\mathrm{COV})\left[X \mid \mathbf{C}_{X}[\kappa]\right] \exists C_{1} \in \mathfrak{C} \exists C_{2} \in \mathfrak{C}: X=C_{1} \cup C_{2}\right) .
$$

In addition, we suppose that

$$
((\mathbb{S} C)-\text { top })[X] \triangleq\{\tau \in(\text { top })[X] \mid((\mathrm{p}, \text { bin })-\mathrm{cl})[X ; \tau] \neq \emptyset\} ;
$$

in addition, $((\mathbb{S C})-$ top $)[X]$ is the family of all supercompact topologies on $X$. Under $\tau \in((\mathbb{S C})-$ top) $[X]$, we obtain supercompact TS $(X, \tau)$; moreover, if $(X, \tau)$ is a $T_{2}$-space, then $(X, \tau)$ is called supercompactum. Every supercompact TS is compact. Then, under $\tau \in((\mathbb{S C})-$ top $)[X]$, in the form of $(X, \tau)$, we obtain (in particular) a compact TS.

\section{Maximal linked systems and ultrafilters: general properties}

In the following, a nonempty set $E$ is fixed. We consider families from $\mathcal{P}^{\prime}(\mathcal{P}(E))$. In addition, we use (1.4)- (1.10).

Filters and ultrafiltres. In the following, we fix $\mathcal{L} \in \pi[E]$ (later, with respect to $\mathcal{L}$, additional conditions will overlap). We consider $(E, \mathcal{L})$ as widely understood measurable space. Then,

$\mathbb{F}^{*}(\mathcal{L}) \triangleq\left\{\mathcal{F} \in \mathcal{P}^{\prime}(\mathcal{L} \backslash\{\emptyset\}) \mid(A \cap B \in \mathcal{F} \forall A \in \mathcal{F} \forall B \in \mathcal{F}) \&(\forall F \in \mathcal{F} \forall L \in \mathcal{L}(F \subset L) \Rightarrow(L \in \mathcal{F}))\right\}$

is the family of all filters of $(E, \mathcal{L})$. Maximal filters are called ultrafilters $(\mathrm{u} / \mathrm{f})$. Then

$$
\begin{gathered}
\mathbb{F}_{0}^{*}(\mathcal{L}) \triangleq\left\{\mathcal{U} \in \mathbb{F}^{*}(\mathcal{L}) \mid \forall \mathcal{F} \in \mathbb{F}^{*}(\mathcal{L}) \quad(\mathcal{U} \subset \mathcal{F}) \Rightarrow(\mathcal{U}=\mathcal{F})\right\}=\left\{\mathcal{U} \in \mathbb{F}^{*}(\mathcal{L}) \mid \forall L \in \mathcal{L}\right. \\
(L \cap U \neq \emptyset \quad \forall U \in \mathcal{U}) \Rightarrow(L \in \mathcal{U})\}=\{\mathcal{U} \in(\text { Cen })[\mathcal{L}] \mid \forall \mathcal{V} \in(\text { Cen })[\mathcal{L}] \quad(\mathcal{U} \subset \mathcal{V}) \Rightarrow(\mathcal{U}=\mathcal{V})\}
\end{gathered}
$$

is the nonempty family of all $\mathrm{u} / \mathrm{f}$ of $(E, \mathcal{L})$. If $x \in E$, then

$$
(\mathcal{L}-\operatorname{triv})[x] \triangleq\{L \in \mathcal{L} \mid x \in L\} \in \mathbb{F}^{*}(\mathcal{L})
$$

is trivial (fixed) filter corresponding to the point $x$. It is known $[14,(5.9)]$ that

$$
\left((\mathcal{L}-\operatorname{triv})[x] \in \mathbb{F}_{0}^{*}(\mathcal{L}) \quad \forall x \in E\right) \Leftrightarrow\left(\mathcal{L} \in \widetilde{\pi}^{0}[E]\right) .
$$

We suppose that $\Phi_{\mathcal{L}}(L) \triangleq\left\{\mathcal{U} \in \mathbb{F}_{0}^{*}(\mathcal{L}) \mid L \in \mathcal{U}\right\} \quad \forall L \in \mathcal{L}$. Then, how easy check,

$$
(\mathbb{U F})[E ; \mathcal{L}] \triangleq\left\{\Phi_{\mathcal{L}}(L): L \in \mathcal{L}\right\} \in \pi\left[\mathbb{F}_{0}^{*}(\mathcal{L})\right]
$$

From $(1.11)$ and $(2.4)$, the inclusion $(\mathbb{U F})[E ; \mathcal{L}] \in(\mathrm{BAS})\left[\mathbb{F}_{0}^{*}(\mathcal{L})\right]$ follows. In addition, topology

$$
\mathbf{T}_{\mathcal{L}}^{*}[E] \triangleq\{\cup\}((\mathbb{U F})[E ; \mathcal{L}])=\left\{\mathbb{G} \in \mathcal{P}\left(\mathbb{F}_{0}^{*}(\mathcal{L})\right) \mid \forall \mathcal{U} \in \mathbb{G} \exists U \in \mathcal{U}: \Phi_{\mathcal{L}}(U) \subset \mathbb{G}\right\} \in(\text { top })\left[\mathbb{F}_{0}^{*}(\mathcal{L})\right]
$$

realizes [14] zero-dimensional $T_{2}$-space

$$
\left(\mathbb{F}_{0}^{*}(\mathcal{L}), \mathbf{T}_{\mathcal{L}}^{*}[E]\right)
$$

Everywhere in the future, we suppose that

$$
\mathcal{L} \in(\mathrm{LAT})_{0}[E] .
$$


By (1.5) and (2.6) we obtain that $\Phi_{\mathcal{L}}\left(L_{1} \cup L_{2}\right) \in \mathcal{P}\left(\mathbb{F}_{0}^{*}(\mathcal{L})\right)$ is defined under $L_{1} \in \mathcal{L}$ and $L_{2} \in \mathcal{L}$; in addition [6], $\Phi_{\mathcal{L}}\left(L_{1} \cup L_{2}\right)=\Phi_{\mathcal{L}}\left(L_{1}\right) \cup \Phi_{\mathcal{L}}\left(L_{2}\right)$. And what is more, in our case (under (2.6))

$$
(\mathbb{U F})[E ; \mathcal{L}] \in(\mathrm{LAT})_{0}\left[\mathbb{F}_{0}^{*}(\mathcal{L})\right] .
$$

Remark 2.1. From (2.4) and (2.7), the following singularity is noticcable: for $(\mathbb{U F})[E ; \mathcal{L}]$, properties of $\mathcal{L}$ are repeated. In this connection, we recall $[6,(9.6)]$ :

$$
(\mathcal{L} \in(\operatorname{alg})[E]) \Longrightarrow\left((\mathbb{U F})[E ; \mathcal{L}] \in(\operatorname{alg})\left[\mathbb{F}_{0}^{*}(\mathcal{L})\right]\right)
$$

Returning to general case of (2.6), we note that (see $[6,(6.7)]$ )

$$
(\mathbb{U F})[E ; \mathcal{L}] \in(\mathrm{cl}-\mathrm{BAS})\left[\mathbb{F}_{0}^{*}(\mathcal{L})\right]
$$

(2.8) permit to define yet one topology. Indeed, by (2.8)

$$
\{\cap\}((\mathbb{U F})[E ; \mathcal{L}]) \in(\operatorname{clos})\left[\mathbb{F}_{0}^{*}(\mathcal{L})\right] .
$$

As a corollary, we obtain that

$$
\mathbf{T}_{\mathcal{L}}^{0}[E] \triangleq \mathbf{C}_{\mathbb{F}_{0}^{*}(\mathcal{L})}[\{\cap\}((\mathbb{U F})[E ; \mathcal{L}])] \in(\operatorname{top})\left[\mathbb{F}_{0}^{*}(\mathcal{L})\right] .
$$

In addition, topology $(2.9)$ converts $[6$, Section 6$] \mathbb{F}_{0}^{*}(\mathcal{L})$ in a compact $T_{1}$-space

$$
\left(\mathbb{F}_{0}^{*}(\mathcal{L}), \mathbf{T}_{\mathcal{L}}^{0}[E]\right) .
$$

We consider (2.5) as analog of Stone space and (2.10) as analog of Wallman space (the space of Wallman extension). In addition (see [15, Proposition 4.1])

$$
\mathbf{T}_{\mathcal{L}}^{0}[E] \subset \mathbf{T}_{\mathcal{L}}^{*}[E]
$$

With regard to (2.11), we consider triplet

$$
\left(\mathbb{F}_{0}^{*}(\mathcal{L}), \mathbf{T}_{\mathcal{L}}^{0}[E], \mathbf{T}_{\mathcal{L}}^{*}[E]\right)
$$

as a bitopological space (BTS); in this connection, see [9]. We do not discuss inessential differences with constructions of [9] and follow to above-mentioned interpretation of (2.12). So,

$$
(\mathbb{U F})[E ; \mathcal{L}] \in(\operatorname{BAS})\left[\mathbb{F}_{0}^{*}(\mathcal{L})\right] \cap(\mathrm{cl}-\mathrm{BAS})\left[\mathbb{F}_{0}^{*}(\mathcal{L})\right]
$$

generates BTS (2.12). It is useful to note the important particular case; namely, if $\mathcal{L} \in(\operatorname{alg})[E]$, then (2.5) is a zero-dimensional compactum or rather the Stone space.

Maximal linked systems. Now, we consider the families $(\mathcal{L}-\operatorname{link})[E]$ and $(\mathcal{L}-\operatorname{link})_{0}[E]$. It is obvious that $\mathbb{F}^{*}(\mathcal{L}) \subset(\mathcal{L}-$ link $)[E]$ and

$$
\mathbb{F}_{0}^{*}(\mathcal{L}) \subset(\mathcal{L}-\operatorname{link})_{0}[E]
$$

Moreover, easy to check that

$$
(\mathcal{L}-\text { link })_{0}[E]=\{\mathcal{E} \in(\mathcal{L}-\text { link })[E] \mid \forall L \in \mathcal{L} \quad(L \cap \Sigma \neq \emptyset \quad \forall \Sigma \in \mathcal{E}) \Longrightarrow(L \in \mathcal{E})\}
$$

(we use the maximality property). With employment of the Zorn lemma, we obtain that

$$
\forall \mathcal{E}_{1} \in(\mathcal{L}-\text { link })[E] \exists \mathcal{E}_{2} \in(\mathcal{L}-\text { link })_{0}[E]: \mathcal{E}_{1} \subset \mathcal{E}_{2} .
$$

Finally, we note the following corollary of maximality of MLS: $\forall \mathcal{E} \in(\mathcal{L}-\text { link })_{0}[E] \quad \forall \Sigma \in \mathcal{E} \forall L \in \mathcal{L}$

$$
(\Sigma \subset L) \Longrightarrow(L \in \mathcal{E}) \text {. }
$$

Therefore, we obtain that

$$
E \in \mathcal{E} \quad \forall \mathcal{E} \in(\mathcal{L}-\text { link })_{0}[E]
$$

The property (2.14) is complemented by the following equality:

$$
\mathbb{F}_{0}^{*}(\mathcal{L})=\left\{\mathcal{U} \in(\mathcal{L}-\text { link })_{0}[E] \mid A \cap B \in \mathcal{U} \quad \forall A \in \mathcal{U} \quad \forall B \in \mathcal{U}\right\} \in \mathcal{P}^{\prime}\left((\mathcal{L}-\text { link })_{0}[E]\right) .
$$




\section{Maximal linked systems; topology of the Wallman type}

We recall that $(2.14)-(2.18)$ are fulfilled under $\mathcal{L}=\mathcal{P}(E)$ (the lattice of all subsets of $E$ ). In addition, $($ link $)[E]=(\mathcal{P}(E)-$ link $)[E]$ and $(\text { link })_{0}[E]=(\mathcal{P}(E)-\text { link })_{0}[E]$ (see (1.34)). As variant of (1.36) and (2.15), we obtain that

$$
\begin{gathered}
(\text { link })_{0}[E]=\{\mathcal{E} \in(\text { link })[E] \mid \forall \mathcal{S} \in(\text { link })[E] \quad(\mathcal{E} \subset \mathcal{S}) \Rightarrow(\mathcal{E}=\mathcal{S})\}= \\
=\{\mathcal{E} \in(\text { link })[E] \mid \forall L \in \mathcal{P}(E) \quad(L \cap \Sigma \neq \emptyset \quad \forall \Sigma \in \mathcal{E}) \Rightarrow(L \in \mathcal{E})\}
\end{gathered}
$$

$(\text { link })_{0}[E] \neq \emptyset$. From $(2.18)$, we obtain that

$$
E \in \mathcal{E} \quad \forall \mathcal{E} \in(\text { link })_{0}[E]
$$

By (2.16) we obtain that

$$
\forall \mathcal{E}_{1} \in(\text { link })[E] \exists \mathcal{E}_{2} \in(\text { link })_{0}[E]: \mathcal{E}_{1} \subset \mathcal{E}_{2} .
$$

Now, we return to arbitrary fixed lattice (2.6). Using (3.1), we consider one property of MLS for lattice (2.6). But, at first, we note one simple corollary of Proposition 1.

Proposition 2. The following property takes place:

$$
\mathcal{E} \cap \mathcal{L} \in(\mathcal{L}-\text { link })[E] \quad \forall \mathcal{E} \in(\text { link })_{0}[E] .
$$

P r o o f. Let $\mathcal{S} \in(\text { link })_{0}[E]$. Using (2.6), consider the family $\mathcal{S} \cap \mathcal{L}$. By (1.4), (1.6), (2.6) and (3.2) $E \in \mathcal{S} \cap \mathcal{L}$. So, $\mathcal{S} \cap \mathcal{L} \neq \emptyset$ and by Proposition $1 \mathcal{S} \cap \mathcal{L} \in(\mathcal{L}-$ link $)[E]$.

Proposition 3. If $\mathcal{E} \in(\mathcal{L}-\text { link })_{0}[E]$, then

$$
\exists \mathcal{S} \in(\text { link })_{0}[E]: \mathcal{E}=\mathcal{S} \cap \mathcal{L}
$$

P r o o f. Fix $\mathcal{E} \in(\mathcal{L}-\operatorname{link})_{0}[E]$. Then, in particular, $\mathcal{E} \in(\mathcal{L}-$ link $)[E]$ and $\forall \mathcal{C} \in(\mathcal{L}-$ link $)[E]$

$$
(\mathcal{E} \subset \mathcal{C}) \Longrightarrow(\mathcal{E}=\mathcal{C}) \text {. }
$$

By $(1.35) \mathcal{E} \in($ link $)[E]$ and $\mathcal{E} \subset \mathcal{L}$. Then $($ see $(3.3))$, for some MLS $\mathcal{V} \in(\text { link })_{0}[E]$

$$
\mathcal{E} \subset \mathcal{V}
$$

In addition, by Proposition 2

$$
\mathcal{V} \cap \mathcal{L} \in(\mathcal{L}-\operatorname{link})[E] .
$$

From (3.6), the inclusion $\mathcal{E} \subset \mathcal{V} \cap \mathcal{L}$ is realized. By (3.5) and (3.7) we obtain the equality

$$
\mathcal{E}=\mathcal{V} \cap \mathcal{L}
$$

So, $\mathcal{V} \in(\text { link })_{0}[E]: \mathcal{E}=\mathcal{V} \cap \mathcal{L}$.

We suppose by analogy with $[4,4.10]$ that

$$
(\mathcal{L}-\text { link })^{0}[E \mid L] \triangleq\left\{\mathcal{E} \in(\mathcal{L}-\text { link })_{0}[E] \mid L \in \mathcal{E}\right\} \quad \forall L \in \mathcal{L} .
$$

Of course, we have the following particular cases:

$$
\left((\mathcal{L}-\text { link })^{0}[E \mid \emptyset]=\emptyset\right) \&\left((\mathcal{L}-\operatorname{link})^{0}[E \mid E]=(\mathcal{L}-\operatorname{link})_{0}[E]\right)
$$


(here and later, we follow to [5]). Using (3.8) and (3.9), we obtain that

$$
\mathfrak{C}_{0}^{*}[E ; \mathcal{L}] \triangleq\left\{(\mathcal{L}-\operatorname{link})^{0}[E \mid L]: L \in \mathcal{L}\right\} \in \mathcal{P}^{\prime}\left(\mathcal{P}\left((\mathcal{L}-\operatorname{link})_{0}[E]\right)\right)
$$

in addition, $\emptyset \in \mathfrak{C}_{0}^{*}[E ; \mathcal{L}]$ and $(\mathcal{L}-\text { link })_{0}[E] \in \mathfrak{C}_{0}^{*}[E ; \mathcal{L}]$. The basic properties of the family (3.10) are considered later. Now, we pass to equipment by topology of the Wallman type. For this, we note that

$$
\mathbf{C}_{E}[\mathcal{L}]=\{E \backslash L: L \in \mathcal{L}\} \in(\mathrm{LAT})_{0}[E] .
$$

In the form of (3.11), we obtain the lattice dual with respect to $\mathcal{L}$.

Remark 3.1. We recall (see [5]) that $\mathcal{L}=\mathbf{C}_{E}[\mathcal{L}]$ under $\mathcal{L} \in(\operatorname{alg})[E]$. So, for the particular case, when $(E, \mathcal{L})$ is a measurable space with algebra of sets, the dual lattice $(3.11)$ coincides with $\mathcal{L}$.

Under $\Lambda \in \mathbf{C}_{E}[\mathcal{L}]$, we suppose that

$$
(\mathcal{L}-\text { link })_{\mathrm{op}}^{0}[E \mid \Lambda] \triangleq\left\{\mathcal{E} \in(\mathcal{L}-\operatorname{link})_{0}[E] \mid \exists \Sigma \in \mathcal{E}: \Sigma \subset \Lambda\right\} ;
$$

of course, we can consider that $\Lambda=E \backslash L$, where $L \in \mathcal{L}$. In this connection, we note that

$$
(\mathcal{L}-\operatorname{link})_{\text {op }}^{0}[E \mid E \backslash L]=(\mathcal{L}-\operatorname{link})_{0}[E] \backslash(\mathcal{L}-\operatorname{link})^{0}[E \mid L] \quad \forall L \in \mathcal{L} .
$$

Of course, by (3.11) $\emptyset \in \mathbf{C}_{E}[\mathcal{L}]$ and $E \in \mathbf{C}_{E}[\mathcal{L}]$; in addition,

$$
\left((\mathcal{L}-\operatorname{link})_{\mathrm{op}}^{0}[E \mid \emptyset]=\emptyset\right) \&\left((\mathcal{L}-\operatorname{link})_{\mathrm{op}}^{0}[E \mid E]=(\mathcal{L}-\operatorname{link})_{0}[E]\right) .
$$

As a corollary, we obtain that by statements of Section 1

$$
\mathfrak{C}_{\text {op }}^{0}[E ; \mathcal{L}] \triangleq\left\{(\mathcal{L}-\operatorname{link})_{\text {op }}^{0}[E \mid \Lambda]: \Lambda \in \mathbf{C}_{E}[\mathcal{L}]\right\} \in(\mathrm{p}-\mathrm{BAS})_{\emptyset}\left[(\mathcal{L}-\text { link })_{0}[E]\right] .
$$

As a corollary, in the form of $\{\cap\}_{\sharp}\left(\mathfrak{C}_{\text {op }}^{0}[E ; \mathcal{L}]\right) \in(\text { op }-\mathrm{BAS})_{\emptyset}\left[(\mathcal{L}-\text { link })_{0}[E]\right]$, we obtain an open base and

$$
\mathbb{T}_{0}(E \mid \mathcal{L}) \triangleq\{\cup\}\left(\{\cap\}_{\sharp}\left(\mathfrak{C}_{\mathrm{op}}^{0}[E ; \mathcal{L}]\right)\right) \in(\text { top })\left[(\mathcal{L}-\operatorname{link})_{0}[E]\right] .
$$

So, we have the following TS

$$
\left((\mathcal{L}-\text { link })_{0}[E], \mathbb{T}_{0}(E \mid \mathcal{L})\right) .
$$

Of course, $\{\cap\}_{\sharp}\left(\mathfrak{C}_{\text {op }}^{0}[E ; \mathcal{L}]\right) \in\left(\mathbb{T}_{0}(E \mid \mathcal{L})-\text { BAS }\right)_{0}\left[(\mathcal{L}-\text { link })_{0}[E]\right]$ and, as a corollary,

$$
\mathfrak{C}_{\mathrm{op}}^{0}[E ; \mathcal{L}] \in(\mathrm{p}-\mathrm{BAS})_{\emptyset}^{0}\left[(\mathcal{L}-\operatorname{link})_{0}[E] ; \mathbb{T}_{0}(E \mid \mathcal{L})\right]
$$

In addition, by (3.13) the following equality is realized:

$$
\mathfrak{C}_{0}^{*}[E ; \mathcal{L}]=\mathbf{C}_{(\mathcal{L}-\text { link })_{0}[E]}\left[\mathfrak{C}_{\mathrm{op}}^{0}[E ; \mathcal{L}]\right]
$$

From (3.17) and (3.18), by duality we obtain (see (1.16)) that

$$
\mathfrak{C}_{0}^{*}[E ; \mathcal{L}] \in(\mathrm{p}-\mathrm{BAS})_{\mathrm{cl}}^{0}\left[(\mathcal{L}-\operatorname{link})_{0}[E] ; \mathbb{T}_{0}(E \mid \mathcal{L})\right] .
$$

From (3.17) and (3.19), we have dual construction for TS (3.16). In addition, (3.17) and (3.19) are open and closed subbases of this TS respectively. By (3.18) self these subbases are situated in a duality. Now, we note the statements of [5] connected with supercompactness of TS (3.16). At first, we recall the notion of closed binary subbases. Namely, by (1.38)

$$
\begin{gathered}
((\mathrm{p}, \text { bin })-\mathrm{cl})\left[(\mathcal{L}-\operatorname{link})_{0}[E] ; \mathbb{T}_{0}(E \mid \mathcal{L})\right]=\left\{\mathfrak{L} \in(\mathrm{p}-\mathrm{BAS})_{\mathrm{cl}}^{0}\left[(\mathcal{L}-\operatorname{link})_{0}[E] ; \mathbb{T}_{0}(E \mid \mathcal{L})\right] \mid\right. \\
\left.\bigcap_{\mathbb{L} \in \lambda} \mathbb{L} \neq \emptyset \quad \forall \lambda \in(\mathfrak{L}-\operatorname{link})\left[(\mathcal{L}-\operatorname{link})_{0}[E]\right]\right\}
\end{gathered}
$$


is the family of all closed binary subbases. We recall that by (1.40)

$$
\left(\mathbb{T}_{0}(E \mid \mathcal{L}) \in((\mathbb{S C})-\text { top })\left[(\mathcal{L}-\text { link })_{0}[E]\right]\right) \Leftrightarrow\left(((\mathrm{p}, \text { bin })-\operatorname{cl})\left[(\mathcal{L}-\text { link })_{0}[E] ; \mathbb{T}_{0}(E \mid \mathcal{L})\right] \neq \emptyset\right) .
$$

In [5], the following statement was established: $\mathfrak{C}_{0}^{*}[E ; \mathcal{L}] \in((\mathrm{p}$, bin $)-\mathrm{cl})\left[(\mathcal{L}-\operatorname{link})_{0}[E] ; \mathbb{T}_{0}(E \mid \mathcal{L})\right]$. From (3.21), we obtain that

$$
\mathbb{T}_{0}(E \mid \mathcal{L}) \in((\mathbb{S C})-\text { top })\left[(\mathcal{L}-\text { link })_{0}[E]\right]
$$

So, (3.16) is a supercompact TS. With employment of (1.39), (3.18), and the above-mentioned property of $\mathfrak{C}_{0}^{*}[E ; \mathcal{L}]$, we have the following statement:

$$
\forall \mathcal{C} \in(\mathrm{COV})\left[(\mathcal{L}-\text { link })_{0}[E] \mid \mathfrak{C}_{\text {op }}^{0}[E ; \mathcal{L}]\right] \exists C_{1} \in \mathcal{C} \exists C_{2} \in \mathcal{C}:(\mathcal{L}-\text { link })_{0}[E]=C_{1} \cup C_{2}
$$

Of course, for (3.23), we use the property

$$
\mathfrak{C}_{\text {op }}^{0}[E ; \mathcal{L}]=\mathbf{C}_{(\mathcal{L}-\text { link })_{0}[E]}\left[\mathfrak{C}_{o}^{*}[E ; \mathcal{L}]\right]
$$

(indeed, (3.24) is obvious corollary of (3.18)). We consider (3.15) as a topology of Wallman type. We note two obvious property. Namely, for $\Lambda_{1} \in \mathbf{C}_{E}[\mathcal{L}]$ and $\Lambda_{2} \in \mathbf{C}_{E}[\mathcal{L}]$

$$
\left(\Lambda_{1} \cap \Lambda_{2}=\emptyset\right) \Rightarrow\left((\mathcal{L}-\operatorname{link})_{\mathrm{op}}^{0}\left[E \mid \Lambda_{1}\right] \cap(\mathcal{L}-\operatorname{link})_{\mathrm{op}}^{0}\left[E \mid \Lambda_{2}\right]=\emptyset\right) .
$$

Moreover, we have the following property of isotonicity: under $\Lambda_{1} \in \mathbf{C}_{E}[\mathcal{L}]$ and $\Lambda_{2} \in \mathbf{C}_{E}[\mathcal{L}]$

$$
\left(\Lambda_{1} \subset \Lambda_{2}\right) \Rightarrow\left((\mathcal{L}-\operatorname{link})_{\mathrm{op}}^{0}\left[E \mid \Lambda_{1}\right] \subset(\mathcal{L}-\operatorname{link})_{\mathrm{op}}^{0}\left[E \mid \Lambda_{2}\right]\right) .
$$

Now, we consider the corresponding equipment for the set of $\mathrm{u} / \mathrm{f}$ of the lattice $\mathcal{L}$. For $\Lambda \in \mathbf{C}_{E}[\mathcal{L}]$, we obtain that

$$
\widetilde{\mathbb{F}}_{\mathbf{C}}[\mathcal{L} \mid \Lambda] \triangleq(\mathcal{L}-\operatorname{link})_{\text {op }}^{0}[E \mid \Lambda] \cap \mathbb{F}_{0}^{*}(\mathcal{L})=\left\{\mathcal{U} \in \mathbb{F}_{0}^{*}(\mathcal{L}) \mid \exists U \in \mathcal{U}: U \subset \Lambda\right\} \in \mathcal{P}\left(\mathbb{F}_{0}^{*}(\mathcal{L})\right) .
$$

Of course, $\widetilde{\mathbb{F}}_{\mathbf{C}}[\mathcal{L} \mid E \backslash L]$ is defined under $L \in \mathcal{L}$. It is obvious that

$$
\widetilde{\mathfrak{F}}_{\mathbf{C}}[\mathcal{L}] \triangleq\left\{\widetilde{\mathbb{F}}_{\mathbf{C}}[\mathcal{L} \mid \Lambda]: \Lambda \in \mathbf{C}_{E}[\mathcal{L}]\right\}=\mathbf{C}_{\mathbb{F}_{0}^{*}(\mathcal{L})}[(\mathbb{U F})[E ; \mathcal{L}]]
$$

In (3.26), the following equality is used: namely, under $L \in \mathcal{L}, \widetilde{\mathbb{F}}_{\mathbf{C}}[\mathcal{L} \mid E \backslash L]=\mathbb{F}_{0}^{*}(\mathcal{L}) \backslash \Phi_{\mathcal{L}}(L)$. Using simple corollary of $(2.8)$ and $(3.26)$, we obtain that $\widetilde{\mathfrak{F}}_{\mathbf{C}}[\mathcal{L}] \in(\operatorname{BAS})\left[\mathbb{F}_{0}^{*}(\mathcal{L})\right]$ (see $(1.12)$ ). In addition, by $(1.13),(2.8)$, and $(3.26)$

$$
\mathbf{T}_{\mathcal{L}}^{0}[E]=\{\cup\}\left(\widetilde{\mathfrak{F}}_{\mathbf{C}}[\mathcal{L}]\right)
$$

So, we obtain the following property (see [5]): namely,

$$
\widetilde{\mathfrak{F}}_{\mathbf{C}}[\mathcal{L}] \in\left(\mathbf{T}_{\mathcal{L}}^{0}[E]-\mathrm{BAS}\right)_{0}\left[\mathbb{F}_{0}^{*}(\mathcal{L})\right] .
$$

In (3.27) and (3.28), we have analog of (3.15) and (3.17) respectively; in addition, it is useful to note that $\emptyset=\widetilde{\mathbb{F}}_{\mathbf{C}}[\mathcal{L} \mid \emptyset] \in \widetilde{\mathfrak{F}}_{\mathbf{C}}[\mathcal{L}]$ (we use $(3.11)$ ) and therefore

$$
\widetilde{\mathfrak{F}}_{\mathbf{C}}[\mathcal{L}] \in(\text { op }-\mathrm{BAS})_{\emptyset}\left[\mathbb{F}_{0}^{*}(\mathcal{L})\right] .
$$

On the other hand, by (3.14), (3.25) and (3.26)

$$
\widetilde{\mathfrak{F}}_{\mathbf{C}}[\mathcal{L}]=\left.\mathfrak{C}_{\mathrm{op}}^{0}[E ; \mathcal{L}]\right|_{\mathbb{F}_{0}^{*}(\mathcal{L})} .
$$

From (3.29), we obtain the following statement of [5]: (2.10) is a subspace of TS (3.16). Namely

$$
\mathbf{T}_{\mathcal{L}}^{0}[E]=\left.\mathbb{T}_{0}(E \mid \mathcal{L})\right|_{\mathbb{F}_{0}^{*}(\mathcal{L})} .
$$

As a corollary, we obtain the useful property: the set $\mathbb{F}_{0}^{*}(\mathcal{L})$ is compact in TS (3.16):

$$
\mathbb{F}_{0}^{*}(\mathcal{L}) \in\left(\mathbb{T}_{0}(E \mid \mathcal{L})-\text { comp }\right)\left[(\mathcal{L}-\text { link })_{0}[E]\right]
$$

Now, the following statement is obvious. 
Proposition 4. If (3.16) is $T_{2}$-space, then $\mathbb{F}_{0}^{*}(\mathcal{L})$ is closed in this space:

$$
\mathbb{F}_{0}^{*}(\mathcal{L}) \in \mathbf{C}_{(\mathcal{L}-\text { link })_{0}[E]}\left[\mathbb{T}_{0}(E \mid \mathcal{L})\right]
$$

In connection with Proposition 4, we note the known property concerning to [4, 4.16] (see too [16, p. 65]).

We note that, for every $\mathcal{E} \in(\mathcal{L}-\operatorname{link})_{0}[E]$, the following equality is realized:

$$
\bigcap_{\Sigma \in \mathcal{E}}(\mathcal{L}-\operatorname{link})^{0}[E \mid \Sigma]=\{\mathcal{E}\}
$$

as a corollary, by (3.19) we obtain that

$$
\{\mathcal{E}\} \in \mathbf{C}_{(\mathcal{L}-\text { link })_{0}[E]}\left[\mathbb{T}_{0}(E \mid \mathcal{L})\right] .
$$

So, we have the following statement of [5].

Proposition 5. By (3.16) a supercompact $T_{1}$-space is realized.

Of course, if (3.16) is a $T_{2}$-space, then it is a supercompactum. We note that by the maximality property $\forall \mathcal{E}_{1} \in(\mathcal{L}-\text { link })_{0}[E] \quad \forall \mathcal{E}_{2} \in(\mathcal{L}-\text { link })_{0}[E]$

$$
\left(\mathcal{E}_{1} \neq \mathcal{E}_{2}\right) \Longleftrightarrow\left(\left(\mathcal{E}_{1} \backslash \mathcal{E}_{2} \neq \emptyset\right) \&\left(\mathcal{E}_{2} \backslash \mathcal{E}_{1} \neq \emptyset\right)\right)
$$

Moreover, it is obvious that $\forall \mathcal{E}_{1} \in(\mathcal{L}-\operatorname{link})_{0}[E] \quad \forall \mathcal{E}_{2} \in(\mathcal{L}-\operatorname{link})_{0}[E]$

$$
\left(\mathcal{E}_{1} \neq \mathcal{E}_{2}\right) \Longleftrightarrow\left(\exists \Sigma_{1} \in \mathcal{E}_{1} \quad \exists \Sigma_{2} \in \mathcal{E}_{2}: \Sigma_{1} \cap \Sigma_{2}=\emptyset\right) .
$$

\section{Maximal linked systems as elements of zero-dimensional $T_{2}$-space and bitopological structure}

In this section, we introduce TS analogous to (2.5). Elements of this new TS are MLS. We recall that by $(1.14),(3.8),(3.10)$, and (3.9)

$$
\mathfrak{C}_{0}^{*}[E ; \mathcal{L}] \in(\mathrm{p}-\mathrm{BAS})_{\emptyset}\left[(\mathcal{L}-\operatorname{link})_{0}[E]\right] .
$$

From (4.1), the obvious property $\{\cap\}_{\sharp}\left(\mathfrak{C}_{0}^{*}[E ; \mathcal{L}]\right) \in(\text { op }-\mathrm{BAS})_{\emptyset}\left[(\mathcal{L}-\text { link })_{0}[E]\right]$ follows. As a corollary,

$$
\mathbb{T}_{*}(E \mid \mathcal{L}) \triangleq\{\cup\}\left(\{\cap\}_{\sharp}\left(\mathfrak{C}_{0}^{*}[E ; \mathcal{L}]\right)\right) \in(\text { top })\left[(\mathcal{L}-\text { link })_{0}[E]\right] .
$$

So, by (4.2) we obtain the required TS

$$
\left((\mathcal{L}-\operatorname{link})_{0}[E], \mathbb{T}_{*}(E \mid \mathcal{L})\right) .
$$

For this TS, by (4.2) we have the inclusion

$$
\mathfrak{C}_{0}^{*}[E ; \mathcal{L}] \in(\mathrm{p}-\mathrm{BAS})_{\emptyset}^{0}\left[(\mathcal{L}-\operatorname{link})_{0}[E] ; \mathbb{T}_{*}(E \mid \mathcal{L})\right] .
$$

So, we obtain the following statement

$$
\mathfrak{C}_{0}^{*}[E ; \mathcal{L}] \in(\mathrm{p}-\mathrm{BAS})_{\emptyset}^{0}\left[(\mathcal{L}-\operatorname{link})_{0}[E] ; \mathbb{T}_{*}(E \mid \mathcal{L})\right] \cap((\mathrm{p}, \text { bin })-\operatorname{cl})\left[(\mathcal{L}-\operatorname{link})_{0}[E] ; \mathbb{T}_{0}(E \mid \mathcal{L})\right]
$$

We obtain some analog of $(2.13)$. So, the family $\mathfrak{C}_{0}^{*}[E ; \mathcal{L}] \ll$ serves» both topology $\mathbb{T}_{*}(E \mid \mathcal{L})$ and topology $\mathbb{T}_{0}(E \mid \mathcal{L})$. But, now we focus on consideration of TS (4.3). 
It is easy proved that $\forall L_{1} \in \mathcal{L} \forall L_{2} \in \mathcal{L}$

$$
\left(L_{1} \cap L_{2}=\emptyset\right) \Longleftrightarrow\left((\mathcal{L}-\text { link })^{0}\left[E \mid L_{1}\right] \cap(\mathcal{L}-\text { link })^{0}\left[E \mid L_{2}\right]=\emptyset\right) ;
$$

in (4.5), we use the property analogous to (3.3). We use (4.6) for verification of separability of the TS (4.2). For this, we introduce the next notion: if $\mathcal{E}_{1}$ and $\mathcal{E}_{2}$ are nonempty families, then

$$
(\text { Dis })\left[\mathcal{E}_{1} ; \mathcal{E}_{2}\right] \triangleq\left\{z \in \mathcal{E}_{1} \times \mathcal{E}_{2} \mid \operatorname{pr}_{1}(z) \cap \operatorname{pr}_{2}(z)=\emptyset\right\}
$$

Of course, in (4.7), we can use arbitrary MLS from $(\mathcal{L}-\text { link })_{0}[E]$ as $\mathcal{E}_{1}$ and $\mathcal{E}_{2}$. Then, by (4.6) and (4.7)

$$
\begin{gathered}
(\mathcal{L}-\text { link })^{0}\left[E \mid \operatorname{pr}_{1}(z)\right] \cap(\mathcal{L}-\text { link })^{0}\left[E \mid \operatorname{pr}_{2}(z)\right]=\emptyset \\
\left.\left.\forall \mathcal{E}_{1} \in(\mathcal{L}-\text { link })_{0}[E] \quad \forall \mathcal{E}_{2} \in(\mathcal{L}-\text { link })_{0}[E] \quad \forall z \in(\text { Dis })\right] \mathcal{E}_{1} ; \mathcal{E}_{2}\right]
\end{gathered}
$$

If $(X, \tau)$ is TS and $x \in X$, then $N_{\tau}^{0}(x) \triangleq\{G \in \tau \mid x \in G\}$. We confine ourselves to employment of open neighborhoods. Of course, by (3.10) and (4.2) we obtain the following obvious property: if $\mathcal{E} \in(\mathcal{L}-\text { link })_{0}[E]$ and $\Sigma \in \mathcal{E}$, then

$$
(\mathcal{L}-\operatorname{link})^{0}[E \mid \Sigma] \in N_{\mathbb{T}_{*}(E \mid \mathcal{L})}^{0}(\mathcal{E}) .
$$

We note that by $(3.32)$, for $\mathcal{E}_{1} \in(\mathcal{L}-\text { link })_{0}[E]$ and $\mathcal{E}_{2} \in(\mathcal{L}-\text { link })_{0}[E] \backslash\left\{\mathcal{E}_{1}\right\}$, the property

$$
(\mathrm{Dis})\left[\mathcal{E}_{1} ; \mathcal{E}_{2}\right] \neq \emptyset
$$

is realized (see (4.7)). As a corollary, by (4.8) and (4.9) $\forall \mathcal{E}_{1} \in(\mathcal{L}-\text { link })_{0}[E]$ $\forall \mathcal{E}_{2} \in(\mathcal{L}-\text { link })_{0}[E] \backslash\left\{\mathcal{E}_{1}\right\} \exists \mathbb{G}_{1} \in N_{\mathbb{T}_{*}(E \mid \mathcal{L})}^{0}\left(\mathcal{E}_{1}\right) \quad \exists \mathbb{G}_{2} \in N_{\mathbb{T}_{*}(E \mid \mathcal{L})}^{0}\left(\mathcal{E}_{2}\right):$

$$
\mathbb{G}_{1} \cap \mathbb{G}_{2}=\emptyset .
$$

So, (4.3) is a $T_{2}$-space. Moreover, we note that by $(2.15)$

$$
(\mathcal{L}-\operatorname{link})^{0}[E \mid L]=\left\{\mathcal{E} \in(\mathcal{L}-\text { link })_{0}[E] \mid L \cap \Sigma \neq \emptyset \quad \forall \Sigma \in \mathcal{E}\right\} \quad \forall L \in \mathcal{L} .
$$

On the other hand, from (4.11) the following property (see [5]) is extracted:

$$
(\mathcal{L}-\text { link })^{0}[E \mid L] \in \mathbb{T}_{*}(E \mid \mathcal{L}) \cap \mathbf{C}_{(\mathcal{L}-\text { link })_{0}[E]}\left[\mathbb{T}_{*}(E \mid \mathcal{L})\right] \quad \forall L \in \mathcal{L}
$$

From (3.10) and (4.12), we obtain that

$$
\mathfrak{C}_{0}^{*}[E ; \mathcal{L}] \subset \mathbb{T}_{*}(E \mid \mathcal{L}) \cap \mathbf{C}_{(\mathcal{L}-\text { link })_{0}[E]}\left[\mathbb{T}_{*}(E \mid \mathcal{L})\right]
$$

Using axioms of TS, from (4.13), we obtain that

$$
\{\cap\}_{\sharp}\left(\mathfrak{C}_{0}^{*}[E ; \mathcal{L}]\right) \subset \mathbb{T}_{*}(E \mid \mathcal{L}) \cap \mathbf{C}_{(\mathcal{L}-\text { link })_{0}[E]}\left[\mathbb{T}_{*}(E \mid \mathcal{L})\right],
$$

where $\{\cap\}_{\sharp}\left(\mathfrak{C}_{0}^{*}[E ; \mathcal{L}]\right) \in(\operatorname{BAS})\left[(\mathcal{L}-\text { link })_{0}[E]\right]$ and by $(4.2)$

$$
\{\cap\}_{\sharp}\left(\mathfrak{C}_{0}^{*}[E ; \mathcal{L}]\right) \in\left(\mathbb{T}_{*}(E \mid \mathcal{L})-\operatorname{BAS}\right)_{0}\left[(\mathcal{L}-\operatorname{link})_{0}[E]\right] .
$$

Proposition 6. In the form of (4.3) a zero-dimensional $T_{2}$-space is realized. 
The corresponding proof (see [5]) is immediate combination of (4.10), (4.14), and (4.15) (see [13, $6.2])$.

We note the following obvious property (see (3.8) and definitions of Section 2)

$$
\Phi_{\mathcal{L}}(L)=(\mathcal{L}-\text { link })^{0}[E \mid L] \cap \mathbb{F}_{0}^{*}(\mathcal{L}) \quad \forall L \in \mathcal{L} .
$$

Therefore, by (2.4), (3.10), and (4.16) we obtain the equality

$$
(\mathbb{U F})[E ; \mathcal{L}]=\left.\mathfrak{C}_{0}^{*}[E ; \mathcal{L}]\right|_{\mathbb{F}_{0}^{*}(\mathcal{L})}
$$

As a corollary, the following important property (see [5]) is realized:

$$
\mathbf{T}_{\mathcal{L}}^{*}[E]=\left.\mathbb{T}_{*}(E \mid \mathcal{L})\right|_{\mathbb{F}_{0}^{*}(\mathcal{L})} .
$$

From (4.17), we obtain the next statement: (2.5) is a subspace of the TS (4.3). So, by (3.30) and $(4.17)$

$$
\left(\mathbf{T}_{\mathcal{L}}^{0}[E]=\left.\mathbb{T}_{0}(E \mid \mathcal{L})\right|_{\mathbb{F}_{0}^{*}(\mathcal{L})}\right) \&\left(\mathbf{T}_{\mathcal{L}}^{*}[E]=\left.\mathbb{T}_{*}(E \mid \mathcal{L})\right|_{\mathbb{F}_{0}^{*}(\mathcal{L})}\right)
$$

In (4.18), we have the natural connection for topological equipments of the spaces of MLS and $\mathrm{u} / \mathrm{f}$. In addition, by [5, Proposition 6.5]

$$
\mathbb{T}_{0}(E \mid \mathcal{L}) \subset \mathbb{T}_{*}(E \mid \mathcal{L}) .
$$

So, by (4.19) we obtain the following BTS

$$
\left((\mathcal{L}-\text { link })_{0}[E], \mathbb{T}_{0}(E \mid \mathcal{L}), \mathbb{T}_{*}(E \mid \mathcal{L})\right)
$$

Of course, by (4.18) we can consider BTS (2.12) as a subspace of BTS (4.20).

\section{Ultrafilters of separable lattice of sets}

In present section, we suppose that

$$
\mathcal{L} \in(\mathrm{LAT})_{0}[E] \cap \widetilde{\pi}^{0}[E] .
$$

By (5.1) we obtain the case of separable lattice. Using (2.3) and (5.1), we obtain that

$$
(\mathcal{L}-\operatorname{triv})[x] \in \mathbb{F}_{0}^{*}(\mathcal{L}) \quad \forall x \in E .
$$

By (5.2) we can introduce operator

$$
x \longmapsto(\mathcal{L}-\operatorname{triv})[x]: E \longrightarrow \mathbb{F}_{0}^{*}(\mathcal{L})
$$

denoted by $\left(\mathcal{L}\right.$-triv)[-]. Of course, $(5.3)$ is an immersion of $E$ into $\mathbb{F}_{0}^{*}(\mathcal{L})$. Therefore, we can consider sets-images

$$
(\mathcal{L}-\operatorname{triv})[\cdot]^{1}(A) \triangleq\{(\mathcal{L}-\operatorname{triv})[x]: x \in A\} \in \mathcal{P}\left(\mathbb{F}_{0}^{*}(\mathcal{L})\right) \quad \forall A \in \mathcal{P}(E) .
$$

We note that by (2.11) and (5.4) the inclusions

$$
\operatorname{cl}\left((\mathcal{L}-\operatorname{triv})[\cdot]^{1}(A), \mathbf{T}_{\mathcal{L}}^{*}[E]\right) \subset \operatorname{cl}\left((\mathcal{L}-\operatorname{triv})[\cdot]^{1}(A), \mathbf{T}_{\mathcal{L}}^{0}[E]\right) \quad \forall A \in \mathcal{P}(E)
$$

are realized. By [5, Proposition 6.6] we have the system of equalities

$$
\operatorname{cl}\left((\mathcal{L}-\operatorname{triv})[\cdot]^{1}(L), \mathbf{T}_{\mathcal{L}}^{*}[E]\right)=\operatorname{cl}\left((\mathcal{L}-\operatorname{triv})[\cdot]^{1}(L), \mathbf{T}_{\mathcal{L}}^{0}[E]\right)=\Phi_{\mathcal{L}}(L) \quad \forall L \in \mathcal{L} .
$$


We recall [17, (4.7)] that $\forall L_{1} \in \mathcal{L} \quad \forall L_{2} \in \mathcal{L}$

$$
\left(L_{1} \subset L_{2}\right) \Longleftrightarrow\left(\Phi_{\mathcal{L}}\left(L_{1}\right) \subset \Phi_{\mathcal{L}}\left(L_{2}\right)\right) .
$$

As an obvious corollary, for $L_{1} \in \mathcal{L}$ and $L_{2} \in \mathcal{L}$

$$
\left(L_{1}=L_{2}\right) \Longleftrightarrow\left(\Phi_{\mathcal{L}}\left(L_{1}\right)=\Phi_{\mathcal{L}}\left(L_{2}\right)\right) .
$$

Proposition 7. If $L_{1} \in \mathcal{L}$ and $L_{2} \in \mathcal{L}$, then

$$
\left(L_{1} \subset L_{2}\right) \Longleftrightarrow\left((\mathcal{L}-\operatorname{link})^{0}\left[E \mid L_{1}\right] \subset(\mathcal{L}-\text { link })^{0}\left[E \mid L_{2}\right]\right) .
$$

P r o o f. By $[5,(2.15)]$ we have implication

$$
\left(L_{1} \subset L_{2}\right) \Longrightarrow\left((\mathcal{L}-\operatorname{link})^{0}\left[E \mid L_{1}\right] \subset(\mathcal{L}-\operatorname{link})^{0}\left[E \mid L_{2}\right]\right) .
$$

Let $(\mathcal{L}-\text { link })^{0}\left[E \mid L_{1}\right] \subset(\mathcal{L}-\text { link })^{0}\left[E \mid L_{2}\right]$. We prove that $L_{1} \subset L_{2}$. Indeed, suppose the contrary: let

$$
L_{1} \backslash L_{2} \neq \emptyset .
$$

With employment of $(5.10)$, we choose $x_{*} \in L_{1} \backslash L_{2}$. Then, $(\mathcal{L}-$ triv $)\left[x_{*}\right] \in \mathbb{F}_{0}^{*}(\mathcal{L})$ and, in particular (see (2.14)),

$$
(\mathcal{L}-\operatorname{triv})\left[x_{*}\right] \in(\mathcal{L}-\operatorname{link})_{0}[E] .
$$

In addition, by the choice of $x_{*}$ we obtain (see Section 2) that $L_{1} \in(\mathcal{L}-\operatorname{triv})\left[x_{*}\right]$. Then, by (3.8) and $(5.11)$

$$
(\mathcal{L}-\operatorname{triv})\left[x_{*}\right] \in(\mathcal{L}-\operatorname{link})^{0}\left[E \mid L_{1}\right] .
$$

Therefore, $(\mathcal{L}-\operatorname{triv})\left[x_{*}\right] \in(\mathcal{L}-\operatorname{link})^{0}\left[E \mid L_{2}\right]$ (we use our supposition). Using (3.8), we obtain that $L_{2} \in(\mathcal{L}-\operatorname{triv})\left[x_{*}\right]$ and, as a corollary, $x_{*} \in L_{2}$. But, this inclusion contradicts to the choice of $x_{*}\left(\right.$ recall that $\left.x_{*} \notin L_{2}\right)$. The obtained contradiction proves the required inclusion $L_{1} \subset L_{2}$. So, implication

$$
\left((\mathcal{L}-\text { link })^{0}\left[E \mid L_{1}\right] \subset(\mathcal{L}-\text { link })^{0}\left[E \mid L_{2}\right]\right) \Longrightarrow\left(L_{1} \subset L_{2}\right)
$$

is established. From (5.9) and (5.12), we obtain (5.8).

Corollary 1. If $L_{1} \in \mathcal{L}$ and $L_{2} \in \mathcal{L}$, then

$$
\left(L_{1}=L_{2}\right) \Longleftrightarrow\left((\mathcal{L}-\text { link })^{0}\left[E \mid L_{1}\right]=(\mathcal{L}-\text { link })^{0}\left[E \mid L_{2}\right]\right) .
$$

The corresponding proof is obvious (see Proposition 7). So, mapping

$$
L \longmapsto(\mathcal{L}-\operatorname{link})^{0}[E \mid L]: \mathcal{L} \longrightarrow \mathfrak{C}_{0}^{*}[E ; \mathcal{L}]
$$

is a bijection from $\mathcal{L}$ onto $\mathfrak{C}_{0}^{*}[E ; \mathcal{L}]$ (see $(3.10)$ and Corollary 1$)$. We note that from (5.6) the next density property follows:

$$
\operatorname{cl}\left((\mathcal{L}-\operatorname{triv})[\cdot]^{1}(E), \mathbf{T}_{\mathcal{L}}^{*}[E]\right)=\operatorname{cl}\left((\mathcal{L}-\operatorname{triv})[\cdot]^{1}(E), \mathbf{T}_{\mathcal{L}}^{0}[E]\right)=\mathbb{F}_{0}^{*}(\mathcal{L})
$$

in $(5.13)$, we use the obvious equality $\Phi_{\mathcal{L}}(E)=\mathbb{F}_{0}^{*}(\mathcal{L})$. 


\section{Some additions}

In this sections, at first, we consider questions meaningful of a duality for families $\mathfrak{C}_{0}^{*}[E ; \mathcal{L}]$ and $\mathfrak{C}_{\mathrm{op}}^{0}[E ; \mathcal{L}]$. For this, we recall that (see Section 3)

$$
\mathfrak{C}_{0}^{*}[E ; \mathcal{L}] \in(\mathrm{p}-\mathrm{BAS})_{\mathrm{cl}}^{0}\left[(\mathcal{L}-\operatorname{link})_{0}[E] ; \mathbb{T}_{0}(E \mid \mathcal{L})\right]
$$

As a corollary, by (4.4) and (6.1) we have the property

$$
\mathfrak{C}_{0}^{*}[E ; \mathcal{L}] \in(\mathrm{p}-\mathrm{BAS})_{0}\left[(\mathcal{L}-\text { link })_{0}[E] ; \mathbb{T}_{*}(E \mid \mathcal{L})\right] \cap(\mathrm{p}-\mathrm{BAS})_{\mathrm{cl}}^{0}\left[(\mathcal{L}-\operatorname{link})_{0}[E] ; \mathbb{T}_{0}(E \mid \mathcal{L})\right]
$$

Proposition 8. The family $\mathfrak{C}_{\mathrm{op}}^{0}[E ; \mathcal{L}]$ is a closed subbase of the TS (4.3):

$$
\mathfrak{C}_{\mathrm{op}}^{0}[E ; \mathcal{L}] \in(\mathrm{p}-\mathrm{BAS})_{\mathrm{cl}}^{0}\left[(\mathcal{L}-\operatorname{link})_{0}[E] ; \mathbb{T}_{*}(E \mid \mathcal{L})\right]
$$

P r o o f. We recall (3.24). So, by (4.5) we have the following statement

$$
\mathfrak{C}_{0}^{*}[E ; \mathcal{L}] \in(\mathrm{p}-\mathrm{BAS})_{\emptyset}^{0}\left[(\mathcal{L}-\operatorname{link})_{0}[E] ; \mathbb{T}_{*}(E \mid \mathcal{L})\right]: \mathfrak{C}_{\mathrm{op}}^{0}[E ; \mathcal{L}]=\mathbf{C}_{(\mathcal{L}-\operatorname{link})_{0}[E]}\left[\mathfrak{C}_{0}^{*}[E ; \mathcal{L}]\right]
$$

Then, by (1.16) we obtain (6.3).

From (3.17) and Proposition 8 we have the following property

$$
\mathfrak{C}_{\text {op }}^{0}[E ; \mathcal{L}] \in(\mathrm{p}-\mathrm{BAS})_{0}\left[(\mathcal{L}-\operatorname{link})_{0}[E] ; \mathbb{T}_{0}(E \mid \mathcal{L})\right] \cap(\mathrm{p}-\mathrm{BAS})_{\mathrm{cl}}^{0}\left[(\mathcal{L}-\operatorname{link})_{0}[E] ; \mathbb{T}_{*}(E \mid \mathcal{L})\right]
$$

In (6.2) and (6.4), we obtain a duality of subbases.

\section{Bitopological space of closed ultrafilters and maximal linked systems}

We recall (5.6). Then, by this property the topologies $\mathbf{T}_{\mathcal{L}}^{0}[E]$ and $\mathbf{T}_{\mathcal{L}}^{*}[E]$ are similar (later, we show that in many cases the above-mentioned topologies are equal). But, now we consider the variant of the set lattice for which the above-mentioned topologies differ typically. Namely, we fix $\tau \in(\mathcal{D}-$ top $)[E]$; so, $\tau \in($ top $)[E]$ for which $(E, \tau)$ is a $T_{1}$-space and (in this section) we suppose that

$$
\mathcal{L}=\mathbf{C}_{E}[\tau]
$$

Under $(7.1)$, we call $\mathrm{u} / \mathrm{f}$ of the set $\mathbb{F}_{0}^{*}(\mathcal{L})$ as closed $\mathrm{u} / \mathrm{f}$. Analogously, for MLS of $(\mathcal{L}-\text { link })_{0}[E]$, under (7.1), we use the term closed MLS. In addition, in our case by (5.1) and (7.1)

$$
\mathbf{C}_{E}[\tau] \in(\mathrm{LAT})_{0}[E] \cap \widetilde{\pi}^{0}[E] .
$$

Of course, $\mathbf{C}_{E}[\tau] \in(\mathcal{D}-$ clos $)[E]$. By (7.2) we have the separable lattice (7.1). Indeed, $\{x\} \in \mathbf{C}_{E}[\tau]$ under $x \in E$ (really, by (1.19) $(E, \tau)$ is a $T_{1}$-space). So, in our case, by (2.3) and (5.2)

$$
(\mathcal{L}-\operatorname{triv})[x] \in \mathbb{F}_{0}^{*}\left(\mathbf{C}_{E}[\tau]\right) \quad \forall x \in E .
$$

Of course, by (7.1) and (7.2) we can use statements of Section 5. In particular, by (5.6), (7.1), and

$$
\operatorname{cl}\left(\left(\mathbf{C}_{E}[\tau]-\operatorname{triv}\right)[\cdot]^{1}(F), \mathbf{T}_{\mathbf{C}_{E}[\tau]}^{*}[E]\right)=\operatorname{cl}\left(\left(\mathbf{C}_{E}[\tau]-\operatorname{triv}\right)[\cdot]^{1}(F), \mathbf{T}_{\mathbf{C}_{E}[\tau]}^{0}[E]\right)=\Phi_{\mathbf{C}_{E}[\tau]}(F) \quad \forall F \in \mathbf{C}_{E}[\tau] .
$$


At the same time, we have (see $[5, \S 7]$ ) the property

$$
\operatorname{cl}\left(\left(\mathbf{C}_{E}[\tau]-\operatorname{triv}\right)[\cdot]^{1}(A), \mathbf{T}_{\mathbf{C}_{E}[\tau]}^{0}[E]\right)=\Phi_{\mathbf{C}_{E}[\tau]}(\operatorname{cl}(A, \tau)) \quad \forall A \in \mathcal{P}(E) .
$$

So, by (7.5) the following statement is realized: TS (2.10) «feels» subsets of $E$ accurate to closure. We recall [5, (7.3)]: for $A \in \mathcal{P}(E)$ and $x_{*} \in \operatorname{cl}(A, \tau) \backslash A$

$$
\left(\mathbf{C}_{E}[\tau]-\operatorname{triv}\right)\left[x_{*}\right] \in \operatorname{cl}\left(\left(\mathbf{C}_{E}[\tau]-\operatorname{triv}\right)[\cdot]^{1}(A), \mathbf{T}_{\mathbf{C}_{E}[\tau]}^{0}[E]\right) \backslash \operatorname{cl}\left(\left(\mathbf{C}_{E}[\tau]-\operatorname{triv}\right)[\cdot]^{1}(A), \mathbf{T}_{\mathbf{C}_{E}[\tau]}^{*}[E]\right) .
$$

With employment of $(7.6)$, we obtain (see $[5,(7.4)])$ in our case

$$
\mathbf{C}_{E}[\tau]=\left\{A \in \mathcal{P}(E) \mid \operatorname{cl}\left(\left(\mathbf{C}_{E}[\tau]-\operatorname{triv}\right)[\cdot]^{1}(A), \mathbf{T}_{\mathbf{C}_{E}[\tau]}^{0}[E]\right)=\operatorname{cl}\left(\left(\mathbf{C}_{E}[\tau]-\operatorname{triv}\right)[\cdot]^{1}(A), \mathbf{T}_{\mathbf{C}_{E}[\tau]}^{*}[E]\right)\right\} .
$$

Finally, by [5, Theorem 7.1] we obtain the following implication:

$$
(\tau \neq \mathcal{P}(E)) \Longrightarrow\left(\mathbf{T}_{\mathbf{C}_{E}[\tau]}^{0}[E] \neq \mathbf{T}_{\mathbf{C}_{E}[\tau]}^{*}[E]\right) .
$$

So, for (7.1) and nondiscrete $T_{1}$-space $(E, \tau)$, BTS (2.12) is nondegenerate. From (4.18) and (7.8), we obtain that

$$
(\tau \neq \mathcal{P}(E)) \Longrightarrow\left(\mathbb{T}_{0}\left(E \mid \mathbf{C}_{E}[\tau]\right) \neq \mathbb{T}_{*}\left(E \mid \mathbf{C}_{E}[\tau]\right)\right) .
$$

We use (7.8) and (7.9) in connection with lattices of the family (1.17).

\section{Some particular cases}

In this section, we fix a lattice

$$
\widetilde{\mathcal{L}} \in(\downarrow-\mathrm{LAT})^{0}[E] .
$$

Then, by (1.18) we obtain that $\widetilde{\mathcal{L}} \cup\{E\} \in(\operatorname{clos})[E]$ and (in particular) $\widetilde{\mathcal{L}} \cup\{E\} \in(\mathrm{LAT})_{0}[E]$. In addition,

$$
\tau_{\widetilde{\mathcal{L}}}^{0}[E]=\mathbf{C}_{E}[\widetilde{\mathcal{L}} \cup\{E\}]=\mathbf{C}_{E}[\widetilde{\mathcal{L}}] \cup\{\emptyset\} \in(\mathcal{D}-\text { top })[E]
$$

realizes the following $T_{1}$-space:

$$
\left(E, \tau_{\widetilde{\mathcal{L}}}^{0}[E]\right)
$$

We recall that (see Section 1), for (8.2) and (8.3), the following property takes place: (8.3) is not $T_{2}$-space. From (8.2), we have the equality

$$
\widetilde{\mathcal{L}} \cup\{E\}=\mathbf{C}_{E}\left[\tau_{\widetilde{\mathcal{L}}}^{0}[E]\right]
$$

(see (1.21)). In addition, by (1.22) we obtain that

$$
\tau_{\widetilde{\mathcal{L}}}^{0}[E] \neq \mathcal{P}(E) .
$$

We recall that by $(1.20) \widetilde{\mathcal{L}} \cup\{E\} \in(\mathcal{D}-\operatorname{clos})[E]$. In addition,

$$
\left(\mathbb{T}_{0}(E \mid \widetilde{\mathcal{L}} \cup\{E\}) \in(\text { top })\left[((\widetilde{\mathcal{L}} \cup\{E\})-\text { link })_{0}[E]\right]\right) \&\left(\mathbb{T}_{*}(E \mid \widetilde{\mathcal{L}} \cup\{E\}) \in(\text { top })\left[((\widetilde{\mathcal{L}} \cup\{E\})-\text { link })_{0}[E]\right]\right) .
$$

In the form of the triplet

$$
\left(((\widetilde{\mathcal{L}} \cup\{E\})-\operatorname{link})_{0}[E], \mathbb{T}_{0}(E \mid \widetilde{\mathcal{L}} \cup\{E\}), \mathbb{T}_{*}(E \mid \widetilde{\mathcal{L}} \cup\{E\})\right),
$$

we obtain a BTS. Of course, (8.7) is a variant of BTS (4.20). By (7.9), (8.4), and (8.5) we obtain that

$$
\mathbb{T}_{0}(E \mid \widetilde{\mathcal{L}} \cup\{E\}) \neq \mathbb{T}_{*}(E \mid \widetilde{\mathcal{L}} \cup\{E\}) .
$$


So, by (8.8) the BTS (8.7) is non-degenerate. Moreover, we have topologies

$$
\left(\mathbf{T}_{\widetilde{\mathcal{L}} \cup\{E\}}^{0}[E] \in(\text { top })\left[\mathbb{F}_{0}^{*}(\widetilde{\mathcal{L}} \cup\{E\})\right]\right) \&\left(\mathbf{T}_{\widetilde{\mathcal{L}} \cup\{E\}}^{*}[E] \in(\text { top })\left[\mathbb{F}_{0}^{*}(\widetilde{\mathcal{L}} \cup\{E\})\right]\right) .
$$

In addition, in the form of the triplet

$$
\left(\mathbb{F}_{0}^{*}(\widetilde{\mathcal{L}} \cup\{E\}), \mathbf{T}_{\widetilde{\mathcal{L}} \cup\{E\}}^{0}[E], \mathbf{T}_{\widetilde{\mathcal{L}} \cup\{E\}}^{*}[E]\right)
$$

we have BTS. Of course, (8.9) is a variant of BTS (2.12). By (7.8), (8.4), and (8.5) we obtain that

$$
\mathbf{T}_{\widetilde{\mathcal{L}} \cup\{E\}}^{0}[E] \neq \mathbf{T}_{\widetilde{\mathcal{L}} \cup\{E\}}^{*}[E] .
$$

So, by (8.10) the BTS (8.9) is non-degenerate. We recall that, in Section 1, the concrete examples of the realization of (8.9) and (8.10) were identified (see Examples 1.1-1.4). Now, we consider yet one example of such type.

Example 8.1. Let $\sqsubseteq$ be a direction on the (nonempty) set $E$. So, we consider the case of nonempty directed set $(E, \sqsubseteq)$. Suppose that

$$
(\sqsubseteq-\mathrm{Ma})_{E}[Y] \triangleq\{z \in E \mid y \sqsubseteq z \quad \forall y \in Y\} \quad \forall Y \in \mathcal{P}(E) .
$$

Then $\mathfrak{M}[E ; \sqsubseteq] \triangleq\left\{Y \in \mathcal{P}(E) \mid(\sqsubseteq-\mathrm{Ma})_{E}[Y] \neq \emptyset\right\}$ is the family of all majorized subsets of $E$. Since $E \neq \emptyset$, we have the obvious property $\emptyset \in \mathfrak{M}[E ; \sqsubseteq]$ (moreover, by the choice of $\sqsubseteq$ we obtain that $\{x ; y\} \in \mathfrak{M}[E ; \sqsubseteq] \quad \forall x \in E \quad \forall y \in E)$. From properties of directed sets, the statement $\mathfrak{M}[E ; \sqsubseteq] \in$ $(\mathrm{LAT})[E]$ is realized. It is obvious that $\{x\} \in \mathfrak{M}[E ; \sqsubseteq] \quad \forall x \in E$. Finally,

$$
\bigcap_{\mathcal{H} \in \mathfrak{H}} \mathcal{H} \in \mathfrak{M}[E ; \sqsubseteq] \quad \forall \mathfrak{H} \in \mathcal{P}^{\prime}(\mathfrak{M}[E ; \sqsubseteq]) .
$$

As a corollary, by (1.17) we obtain the implication

$$
(E \notin \mathfrak{M}[E ; \sqsubseteq]) \Longrightarrow\left(\mathfrak{M}[E ; \sqsubseteq] \in(\downarrow-\mathrm{LAT})^{0}[E]\right) .
$$

So, under $E \notin \mathfrak{M}[E ; \sqsubseteq]$, in the form of $\mathfrak{M}[E ; \sqsubseteq]$, we obtain yet one variant of the family of $(\downarrow-\mathrm{LAT})^{0}[E]: \mathfrak{M}[E ; \sqsubseteq] \in(\downarrow-\mathrm{LAT})^{0}[E]$.

\section{Measurable space with algebra of sets}

Recall that by (1.6) and (1.7) $(\mathrm{alg})[E] \subset(\mathrm{LAT})_{0}[E]$. Using this property, in the present section, we consider the case

$$
\mathcal{L} \in(\operatorname{alg})[E] .
$$

By $(9.1)$ we have that (in the present section) $(E, \mathcal{L})$ is a measurable space with algebra of sets. We recall Remark 2.1: in the form of

$$
\left(\mathbb{F}_{0}^{*}(\mathcal{L}),(\mathbb{U F})[E ; \mathcal{L}]\right)
$$

a measurable space with algebra of sets is realized also. Moreover, we have BTS (2.12). But, by [6, Proposition 9.2] this BTS is degenerate:

$$
\mathbf{T}_{\mathcal{L}}^{0}[E]=\mathbf{T}_{\mathcal{L}}^{*}[E]
$$

By (9.2) we obtain the following equality of TS:

$$
\left(\mathbb{F}_{0}^{*}(\mathcal{L}), \mathbf{T}_{\mathcal{L}}^{0}[E]\right)=\left(\mathbb{F}_{0}^{*}(\mathcal{L}), \mathbf{T}_{\mathcal{L}}^{*}[E]\right)
$$


of course, (9.3) is a nonempty zero-dimensional compactum. Moreover, by (9.1)

$$
\mathcal{L}=\mathbf{C}_{E}[\mathcal{L}] .
$$

Therefore, the sets $(\mathcal{L}-\operatorname{link})_{\text {op }}^{0}[E \mid L], L \in \mathcal{L}$, are defined. In addition,

$$
(\mathcal{L}-\text { link })_{\text {op }}^{0}[E \mid L]=(\mathcal{L}-\text { link })^{0}[E \mid L]
$$

under $L \in \mathcal{L}$. We recall that, under $\Lambda \in \mathcal{L}$, the inclusion $E \backslash \Lambda \in \mathcal{L}$ is realized; and what is more, by $(3.13)$

$$
(\mathcal{L}-\operatorname{link})^{0}[E \mid E \backslash \Lambda]=(\mathcal{L}-\operatorname{link})_{0}[E] \backslash(\mathcal{L}-\operatorname{link})^{0}[E \mid \Lambda] .
$$

As a simple corollary, in our case, the equality

$$
\mathfrak{C}_{0}^{*}[E ; \mathcal{L}]=\mathfrak{C}_{\mathrm{op}}^{0}[E ; \mathcal{L}]
$$

is realized. Therefore, by (3.15) and (4.2)

$$
\mathbb{T}_{0}(E \mid \mathcal{L})=\mathbb{T}_{*}(E \mid \mathcal{L}) .
$$

So, by (9.5) we have the following important property: TS

$$
\left((\mathcal{L}-\text { link })_{0}[E], \mathbb{T}_{0}(E \mid \mathcal{L})\right)=\left((\mathcal{L}-\text { link })_{0}[E], \mathbb{T}_{*}(E \mid \mathcal{L})\right)
$$

is a nonempty supercompactum. In particular, (9.6) is a nonempty compactum.

Proposition 9. The set $\mathbb{F}_{0}^{*}(\mathcal{L})$ is closed in $T S(9.6)$ :

$$
\mathbb{F}_{0}^{*}(\mathcal{L}) \in \mathbf{C}_{(\mathcal{L}-\text { link })_{0}[E]}\left[\mathbb{T}_{0}(E \mid \mathcal{L})\right]
$$

The corresponding proof follows from Proposition 4 (indeed, for (9.6) we have the separability property). In connection with Proposition 9, we recall (3.31).

\section{Open maximal linked systems}

In this section, we suppose that

$$
\mathcal{L}=\tau
$$

where $\tau \in($ top $)[E]$. So, $(E, \mathcal{L})=(E, \tau)$ is a TS. We consider the lattice of open sets. In this connection, we recall (see $[15$, Section 8$]$ ) that

$$
\mathbf{T}_{\tau}^{0}[E]=\mathbf{T}_{\tau}^{*}[E] .
$$

Of course, in the form of

$$
\left(\mathbb{F}_{0}^{*}(\tau), \mathbf{T}_{\tau}^{0}[E]\right)=\left(\mathbb{F}_{0}^{*}(\tau), \mathbf{T}_{\tau}^{*}[E]\right),
$$

we obtain a nonempty zero-dimensional compactum of open $\mathrm{u} / \mathrm{f}$ (using (10.1), we consider $\mathrm{u} / \mathrm{f}$ consisting of open sets as open $\mathrm{u} / \mathrm{f}$ ). On the other hand, by (10.1) we can consider MLS consisting of open sets. We call such MLS open also (recall that (top) $\left.[E] \subset(\operatorname{LAT})_{0}[E]\right)$. By $[5$, Proposition 9.1]

$$
(\tau-\text { link })_{0}[E] \backslash(\tau-\operatorname{link})^{0}[E \mid G]=(\tau-\text { link })^{0}[E \mid E \backslash \operatorname{cl}(G, \tau)] \quad \forall G \in \tau .
$$

With employment of this property, in [5, Proposition 9.2], the equality

$$
\mathbb{T}_{0}(E \mid \tau)=\mathbb{T}_{*}(E \mid \tau)
$$


was established. By (10.4) we obtain that

$$
\left((\tau-\operatorname{link})_{0}[E], \mathbb{T}_{0}(E \mid \tau)\right)=\left((\tau-\operatorname{link})_{0}[E], \mathbb{T}_{*}(E \mid \tau)\right)
$$

is a zero-dimensional supercompactum. In addition, by Proposition 4

$$
\mathbb{F}_{0}^{*}(\tau) \in \mathbf{C}_{(\tau-\operatorname{link})_{0}[E]}\left[\mathbb{T}_{0}(E \mid \tau)\right] ;
$$

so, $\mathbb{F}_{0}^{*}(\tau)$ is the closed in the supercompactum (10.5). We obtain that compactum (10.3) is a closed subspace of the supercompactum (10.5).

\section{Conclusion}

We reviewed two BTS. In the first case point of BTS are MLS and, in the second case, similar points are $\mathrm{u} / \mathrm{f}$ of a set lattice. It is established that the second BTS can be considered as a subspace of the first BTS. We indicated the natural variants of our lattice for which the above-mentioned BTS are degenerate and, opposite, the variants with degeneracy of the corresponding BTS is absent. Our consideration is connected with ideas of supercompactness and superextension of a TS. For degenerate BTS the corresponding space of MLS is a supercompactum. Under consideration of the lattice of closed MLS, we obtain a non-degenerate BTS typically.

\section{REFERENCES}

1. De Groot J. Superextensions and supercompactness // Proc. I. Intern. Symp. on extension theory of topological structures and its applications. Berlin: VEB Deutscher Verlag Wis., 1969. P. 89-90.

2. Van Mill J. Supercompactness and Wallman spaces. Amsterdam. Math. Center Tract., 85. 1977.

3. Strok M. and Szymanski. Compact metric spaces have binary bases // Fund. Math., 1975. Vol. 89. P. 81-91.

4. Fedorchuk V.V., Filippov V.V. Obshhaya topologiya. Osnovnyie konstrukzii. M.: Fismatlit, 2006. [in Russian]

5. Chentsov A.G. Ultrafilters and maximal linked systems of sets // Vestn. Udmurtsk. Univ. Mat. Mekh. Komp. Nauki, 2017. Vol. 27, no. 3. P. 365-388. [in Russian]

6. Chentsov A.G. Filters and ultrafilters in the constructions of attraction sets // Vestn. Udmurtsk. Univ. Mat. Mekh. Komp. Nauki, 2011. No. 1, P. 113-142. [in Russian]

7. Chentsov A.G. Tier mappings and ultrafilter-based transformations // Trudy Inst. Mat. i Mekh. UrO RAN, 2012. Vol. 18, no. 4. P. 298-314. [in Russian]

8. Chentsov A.G. Compactifiers in extension constructions for reachability problems with constraints of asymptotic nature // Steklov Inst. Math. 2017. Vol. 296, suppl. 1. P. 102-118. DOI: 10.1134/S0081543817020109

9. Dvalishvili B.P. Bitopological Spaces: Theory, Relations with Generalized Algebraic Structures, and Applications. Nort-Holland. Mathematics studies. 2005.

10. Kuratowski K., Mostowski A. Set theory. Amserdam: North-Holland, 1967.

11. Alexanfroff P.S. Vvedenie v teoriyu mnogestv i obshhuju topologiyu. M.: Editorial URSS, 2004. [in Russian]

12. Alexandroff A.D. Additive set-functions in abstract spaces // Mathematics of the USSR-Sbornik. 1940. Vol. 8, no. 2. P. 307-348.

13. Engelking R. General topology. Warsaw: PWN. 1977.

14. Chentsov A.G. Attraction sets in abstract attainability problems: equivalent representations and basic properties // Russ Math., 2013. Vol. 57, no. 28. DOI:10.3103/S1066369X13110030

15. Chentsov A.G., Pytkeev E.G. Some topological structures of extensions of abstract reachability problems // Proc. Steklov Inst. Math., 2016. 292, suppl. 1. P. 36-54. DOI: 10.1134/S0081543816020048

16. Chentsov A.G. Superextension as bitopological space // Izv. IMI UdGU, 2017. Vol. 49. P. 55-79. [in Russian]

17. Chentsov A.G. To the validity of constraints in the class of generalized elements // Vestn. Udmurtsk. Univ. Mat. Mekh. Komp. Nauki, 2014. Vol. 3. P. 90-109. [in Russian] 